\title{
A 25-Intersection Model for Representing Topological Relations between Simple Spatial Objects in 3-D Space
}

\author{
Mengyun Zhou and Qingfeng Guan * \\ School of Geography and Information Engineering, China University of Geosciences, No.388 Lumo Road, \\ Wuhan 430074, China; myzhou@cug.edu.cn \\ * Correspondence: guanqf@cug.edu.cn
}

Received: 1 March 2019; Accepted: 4 April 2019; Published: 7 April 2019

\begin{abstract}
With the rapid development of the economy, urgent needs for 3-D Geographical Information System (GIS) have sprung up in many application fields. The precise expression of three-dimensional topological relations is the foundation of spatial analysis, topological query, and spatial reasoning in three-dimensional space. In this paper, we subdivide the topological part "boundary" into face, edge, and vertex and propose a 25-intersection model (25IM) to represent topological relations between two simple spatial objects (point, line, region, and body) in 3-D space. An object in the 25IM has five topological parts: vertex, edge, face, interior, and exterior. The classification of topological relations is simplified by merging contain/inside and cover/coveredby. The 25IM describes ten groups of topological relations: body/body, body/region, body/line, body/point, region/region, region/line, region/point, line/line, line/point, and point/point. The 25IM is demonstrated to be more expressive than the 9IM and the DE-9IM, especially in distinguishing the detail situations when one object meets/covers another object (e.g., two bodies meet/cover at vertex, edge, or face).
\end{abstract}

Keywords: Topological Relation; 25-intersection model; 3-D topology; geometric primitive

\section{Introduction}

The spatial relations usually fall into three types: topological relations, direction relations, and metric relations [1,2]. Among them, topological relations are the primary information [3]. The topological relations are the foundation of spatial analysis, topological query, and spatial reasoning as a basic spatial relation. The topological relations are effective for spatial querying and human decision-making [4].

Egenhofer and Franzosa proposed the 4-intersection model (4IM) based on the point set topology theory in 1991 [5]. The 4IM divides the spatial object into boundary and interior and describes the topological relations between two spatial objects using a quaternion matrix represented by Equation (1), where $\partial \mathrm{A}, A^{0}, \partial \mathrm{B}$, and $B^{o}$ denote $\mathrm{A}^{\prime}$ s boundary, A's interior, B's boundary, and B's interior, respectively. The 4IM can distinguish 16 topological relations by taking the element values as empty or non-empty. The 4IM has a limited ability to distinguish topological relations because it is easy to confuse line/line relations and line/region relations.

$$
R_{4 I M}(A, B)=\left[\begin{array}{ll}
A^{o} \cap B^{o} & A^{o} \cap \partial \mathrm{B} \\
\partial \mathrm{A} \cap B^{o} & \partial \mathrm{A} \cap \partial \mathrm{B}
\end{array}\right]
$$

Aiming at solving this problem of 4IM, Egenhofer and Herring introduced the complementary set $A^{-}$as the exterior of the object and extended the 4IM to the 9-intersection model (9IM) [6]. The 9IM describes the topological relations between two spatial objects using a $3 \times 3$ matrix represented 
by Equation (2), where $\partial \mathrm{A}, A^{o}, A^{-}, \partial \mathrm{B}, B^{o}$, and $B^{-}$denote $\mathrm{A}^{\prime}$ s boundary, $\mathrm{A}^{\prime}$ s interior, $\mathrm{A}^{\prime}$ s exterior, $\mathrm{B}^{\prime} \mathrm{s}$ boundary, B's interior, and B's exterior, respectively. A spatial object in the 9IM is a point, a simple line without self-intersection, or a simple region with a connected interior (Figure 1). The 9IM can distinguish $2^{9}=512$ topological relations by taking the element values as empty or non-empty. These topological relations can be grouped into eight kinds: disjoint, contain, inside, equal, meet, cover, coveredby, and overlap. The 9IM is commonly used to express the topological relations between two objects in two-dimensional space because of its concise expression form and strong expression ability. However, the 9IM still has limitations. For example, the 9IM cannot distinguish whether two objects share a common vertex, edge, or face when one body meets or covers another body.

$$
R_{9 I M}(A, B)=\left[\begin{array}{ccc}
A^{o} \cap B^{o} & A^{o} \cap \partial \mathrm{B} & A^{o} \cap B^{-} \\
\partial \mathrm{A} \cap B^{o} & \partial \mathrm{A} \cap \partial \mathrm{B} & \partial \mathrm{A} \cap B^{-} \\
A^{-} \cap B^{o} & A^{-} \cap \partial \mathrm{B} & \partial \mathrm{B} \cap B^{-}
\end{array}\right],
$$

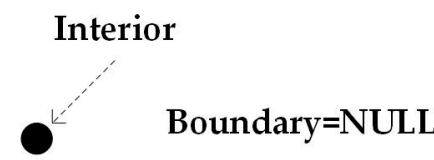

Exterior

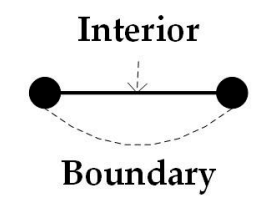

Exterior

(b) Line

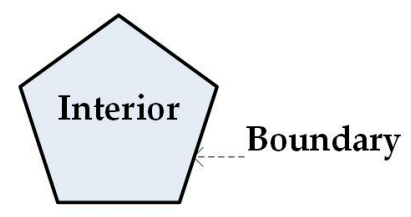

Exterior

(c) Region

(a) Point

Figure 1. The structure of spatial objects in a 9-intersection model (9IM).

The dimensional extended 9-intersection model (DE-9IM) extended the 9IM by considering the dimensions of the intersections (dim(S)) as shown in Equation (3) [7]. The DE-9IM can distinguish more kinds of topological relations than the 9IM and has been used as a basis for query standards in spatial databases (e.g., Post GIS and SQLServer). However, the DE-9IM only records the highest dimensions of the intersections and cannot tell whether there are intersections of lower dimensions.

$$
\operatorname{dim}(S)=\left\{\begin{array}{l}
-1, \text { if } S=\varnothing \\
0, \text { if } S \text { contains at least a point and no lines or areas } \\
1, \text { if } S \text { contains at least a line and no areas } \\
2, \text { if } S \text { contains at least an area }
\end{array},\right.
$$

In recent years, many other models expressing the topological relations in 2-D space and spherical space have been proposed by extending the 4IM and 9IM. The multilevel formal model described all the necessary details of region/region topological relations using three levels of topological invariant [8]. Liu and Shi expressed the topological relations between two GIS objects using an infinite sequence of matrices by considering more topological properties including connectivity and the first fundamental group [9]. In 2008, Yohei Kurata proposed a 9+-intersection model (9+IM) for expressing the topological relations between two complex objects with multiple holes by considering the relations between the subparts of the three topological parts of objects A and B [10]. The uncertain intersection and difference model was proposed to express topological relations between uncertain spatial regions [11]. The 27-intersection model (27IM) was proposed taking both the dimension of intersections and the number of separations into account [12]. The 16-intersection model (16IM) was proposed to describe the topological relations between spherical regions with holes [13].

The theory and technology of two-dimensional (2-D) topological relations have been well studied and applied in commercial software such as ArcGIS. They have been widely used in various fields of physical geography, such as land use analysis and hydrological analysis. With the rapid development 
of the economy, urgent needs for 3-D GIS have sprung up in many application fields, such as urban planning, real estate, flood disaster analysis, air pollution analysis, geological disasters, noise prediction, environmental monitoring, communication base station settings, indoor navigation, mining engineering, and underground excavation. There are many application requirements involving elevation, such as "how far is the groundwater from the land surface?", "what rock formation is under the oil resources?", and "how deep is required to drill?", etc. These questions cannot be solved in 2-D GIS due to the lack of elevation, forcing researchers to study 3-D GIS and 3-D topologies. The precise expression of three-dimensional topological relations is the foundation of spatial analysis and spatial reasoning in three-dimensional space, such as mining route calculations and flood modelling and analysis.

Topological relations between spatial objects in three-dimensional space were initially investigated within the framework of the 9IM $[3,10,14,15]$. However, the topological relations between two objects in the three-dimensional space are more complicated than those in two-dimensional space and cannot be expressed in detail by the 9IM. Among the eight basic topological relations (disjoint, meet, overlap, covers, contains, coveredby, inside, and equal), the cases of meet, cover, and coveredby in three-dimensional space are different from those in two-dimensional space. The intersection can be point, line, and region. The 9IM expressions corresponding to the three different cases in Figure 2 are the same. Although the DE-9IM and its extensions can record the highest dimensions of the intersections, they cannot distinguish the precise conditions. For example, the DE-9IM cannot distinguish whether $A$ and $B$ share a common vertex, edge, or face or whether just a face of $B$ touches a face of $A$ when $\operatorname{dim}(\partial \mathrm{A} \cap \partial \mathrm{B})=2$. As shown in Section 4, the DE-9IM expressions corresponding to the three different cases in Figure 3 are the same. Thus, the 9IM and the DE-9IM cannot satisfy the precise expression of the topological relations between two simple objects in three-dimensional space.

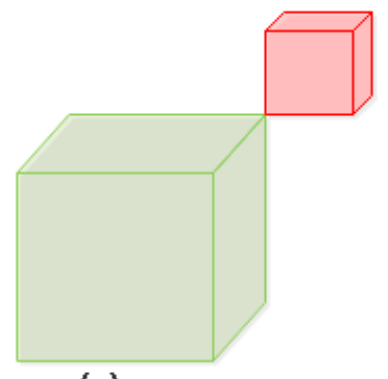

(a)

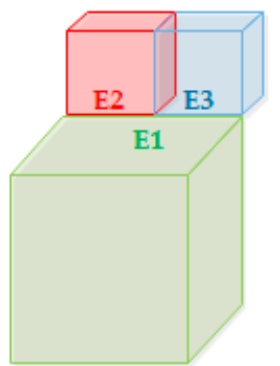

(b)

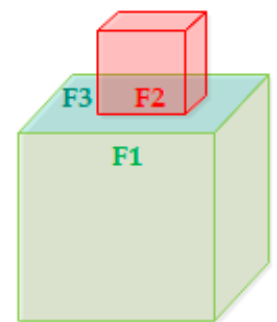

(c)

Figure 2. Different cases of body/body meet relations: (a) A and B meet at a vertex, (b) A and B meet at an edge, and (c) A and B meet at a face. Spatial object A is green, spatial object B is red, and spatial object $C$ is blue in this figure and the following figures. E1, E2, E3, F1, F2, and F3 (F3 = F1 - F2) denotes edge 1 , edge 2 , edge 3 , face 1 , face 2 , and face 3 , respectively.

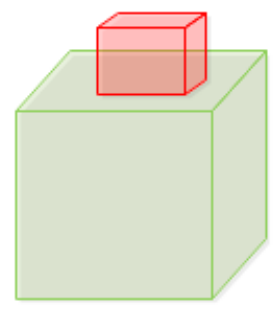

(a)

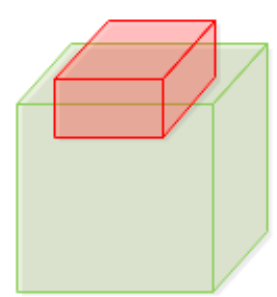

(b)

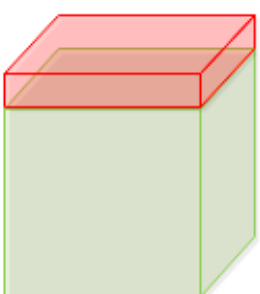

(c)

Figure 3. Body/body meet at face: (a) A's face contains B's face, (b) A's face overlaps B's face, (c) A's face is equal to B's face.

In the real world, it is often required to calculate the common area in calculating property rights. In the 3-D cadastral systems, it is essential to confirm the ownership of each parcel body, boundary face, boundary line, and boundary point. In order to maintain the consistency of topological relations 
and property rights in the database, a face/edge must be divided at the boundaries of parcel bodies when it crosses two parcel bodies. As shown in Figure 2b, parcel bodies B and C meet parcel body A at E1; then, E1 must be divided into E2 and E3. Thus, A's edges are E2, E3, and its other 11 original edges. As shown in Figure 2c, parcel body B meet parcel body A at F2; then, F1 must be divided into F2 and F3. Thus, A's faces are F2, F3, and its other 5 original faces. No operation is required for Figure 2a. It is not enough just to know whether A meets $B$ when there is a command to merge $A$ and $B$. A meets $B$ in all cases of Figure 2, but only A and B in Figure 2c can be merged. Therefore, how to differentiate the precise conditions of intersections should be a focus of study in the topological relations between objects in three-dimensional space.

In the real world, there are rare cases where the boundaries of objects are smooth arcs, and most objects are angular. Vertex, edge, and face are the basic graphic primitives of 3-D spatial data models. Thus, we subdivide the boundary into face, edge, and vertex and propose a 25 -intersection model (25IM) to express the topological relations between two simple objects (point, line, region, and body) in the 3-D space. An object in the 25IM has five topological parts: vertex, edge, face, interior, and exterior. The 25IM describes the topological relations between two spatial objects using a $5 \times 5$ matrix and will be explained in detail in Section 2.2. Excluding the nonexistent cases in the real world, the 25IM can totally distinguish 6259 relations: 2651 relations between two bodies, 2588 relations between a body and a region, 368 relations between a body and a line, 10 relations between a body and a point, 497 relations between two regions, 110 relations between a region and a line, 8 relations between a region and a point, 19 relations between two lines, 6 relations between a line and a point, and 2 relations between two points.

The 25IM can distinguish the detail situations when one object meets/covers another object (e.g., two objects share a common vertex, edge, or face). As shown in Section 4, the 25IM is more expressive than the 9IM and DE-9IM.

\section{Methodology}

The implementation of topological relations is affected by several factors, such as the definition of the spatial objects, the dimension of the space, and the topological relation model. This paper proposes a 25IM for expressing topological relations between two simple objects in 3-D space. This section defines the spatial objects in the 3-D space and lists the topological relations that exist in the real world. Only simple objects are discussed in this paper. Simple lines are lines without a self-intersection and branch. Simple regions are surfaces with a connected interior and without holes. Simple bodies are three-dimensional counterparts of simple regions.

\subsection{Definition of Spatial Objects in Three-Dimensional Space}

The definition of spatial objects is the foundation of topological relations. Topological relations may have significant differences based on different definitions of spatial objects. Therefore, spatial objects must be defined first to study topological relations. We define spatial objects in three-dimensional space and their topological parts differently from most existing models.

In the existing models, the interior, the edge, and the exterior of the point are 0-D, empty, and 3-D, respectively. The interior, the edge, and the exterior of the line are 1-D, 0-D, and 3-D, respectively. The interior, the edge, and the exterior of the region are 2-D, 1-D, and 3-D, respectively. The interior, the edge, and the exterior of the body are 3-D, 2-D, and 3-D, respectively. However, a line in 3-D space has an empty interior $[9,11]$.

Proof 1. Let $\mathrm{A}$ be a set in 3-D space and let $x \in A$; $\mathrm{x}$ is then called an interior element of $\mathrm{A}$ if there is a small open sphere $\mathrm{S}(x, r) \subset A$. A's interior in 3-D space is defined as the collection of all A's interior elements. If we pick up an arbitrary point in a line, even the smallest open sphere with this point as the center will contain some points outside the line. Thus, according to the definition of interior, a line in 3-D space has an empty interior. 
Similarly, a point in 3-D space has an empty interior, and a region in 3-D space also has an empty interior. This fact is in contradiction with the definition of spatial objects in most existing models. Therefore, we define the spatial objects in 3-D space in a different way. In addition, only the exterior of spatial objects is always 3-D in existing models; the interior and boundary of spatial objects are uncertain in dimension. In order to maintain the dimensional consistency of a topological part and to distinguish more different topological relations in details, the 25IM refines the 9IM by subdividing the boundary into face, edge, and vertex. Thus, a spatial object has five topological parts: exterior (3-D), interior (3-D), face (2-D), edge (1-D), and vertex (0-D).

Theorem 1. Except for the exterior, any topological part with a higher dimension than the spatial object is empty. For example, a point (0-D) has an empty edge (1-D), face (2-D), and interior (3-D) and a non-empty vertex (0-D) and exterior (3-D).

Points, lines, regions, and bodies are defined as follows.

A point is a 0-dimensional geometric object and represents a single location in coordinate space (Figure 4a). The vertex of a point is the point self. The exterior of a point includes everything that is not the point. The edge, face, and interior of a point are empty.

A line is a 1-dimensional geometric object usually stored as a sequence of points with linear interpolation between the vertices (Figure $4 \mathrm{~b}$ ). A simple line never passes through the same point twice. The edge of a line is itself without its endpoints. The vertices of a line are its endpoints. The exterior of a line includes everything that is not the line. The interior and face of a line are empty.

A region is a 2-dimensional geometric object enclosed by a boundary line (Figure 4c). The face of a region is the region without its edges and vertices. The edges of a region are the lines forming the region, without their endpoints. The vertices of a region are the endpoints of the edges. The exterior of a region includes everything that is not the region. The interior of a region is empty.

A body is a 3-dimensional geometric object enclosed by its surface (Figure $4 \mathrm{~d}$ ). The interior of a body is the 3-D space enclosed by its boundaries. The faces of a body are the surfaces without their edges. The edges of a body are the lines without their endpoints. The vertices of a body are the endpoints of the edges. The exterior of a body includes everything that is not the body.

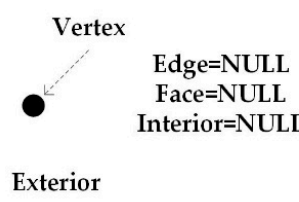

(a) Point

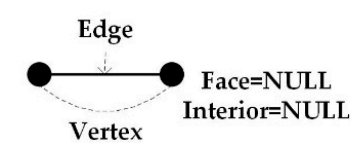

Exterior

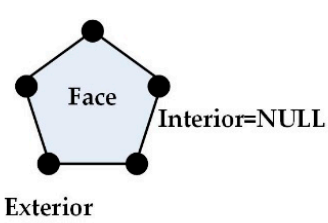

(c) Region

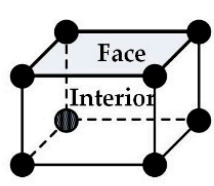

Exterior

(d) Body

Figure 4. A diagram of spatial objects with vertex, edge, face, interior, and exterior.

Based on the definition of spatial objects, ten groups of topological relations can be expressed. These groups are body/body, body/region, body/line, body/point, region/region, region/line, region/point, line/line, line/point, and point/point relations, and they will be described in Section 3.

\subsection{5-Intersection Model}

The 25-intersection model (25IM) describes the topological relations between spatial object $\mathrm{A}$ and spatial object $B$ using a $5 \times 5$ matrix represented by the following formula:

$$
R_{25 I M}(A, B)=\left[\begin{array}{lllll}
A^{v} \cap B^{v} & A^{v} \cap B^{e} & A^{v} \cap B^{f} & A^{v} \cap B^{o} & A^{v} \cap B^{-} \\
A^{e} \cap B^{v} & A^{e} \cap B^{e} & A^{e} \cap B^{f} & A^{e} \cap B^{o} & A^{e} \cap B^{-} \\
A^{f} \cap B^{v} & A^{f} \cap B^{e} & A^{f} \cap B^{f} & A^{f} \cap B^{o} & A^{f} \cap B^{-} \\
A^{o} \cap B^{v} & A^{o} \cap B^{e} & A^{o} \cap B^{f} & A^{o} \cap B^{o} & A^{o} \cap B^{-} \\
A^{-} \cap B^{v} & A^{-} \cap B^{e} & A^{-} \cap B^{f} & A^{-} \cap B^{o} & A^{-} \cap B^{-}
\end{array}\right],
$$


The formula is defined as follows:

1. Let $\mathrm{Q}$ be the universal set, $\mathrm{A} \subset \mathrm{Q}$ and $\mathrm{B} \subset \mathrm{Q}$.

2. A's vertex, edge, face, interior, and exterior are represented by $A^{v}, A^{e}, A^{f}, A^{o}$, and $A^{-}$, respectively.

3. A's boundary $\partial \mathrm{A}=A^{v}+A^{e}+A^{f}, \mathrm{~A}^{\prime}$ s closure $\bar{A}=A^{v}+A^{e}+A^{f}+A^{o}$, and $\mathrm{A}^{\prime}$ s exterior $A^{-}=Q-\bar{A}$.

4. The exterior $A^{-}$, interior $A^{o}$, face $A^{f}$ and edge $A^{e}$ are open sets, and the vertex set $A^{v}$ is a closed set.

5. The value of an intersection is 0 or 1 , where 0 denotes that the intersection is empty, and 1 denotes that the intersection is non-empty.

Theoretically, there are $2^{25}=33554432$ topological relations between spatial objects in 3-D space which can be distinguished based on the 25IM, by considering the values empty and non-empty. However, some of the topological relations do not exist in the real world. The existing topological relations will be discussed in Section 3.

\section{Results}

The types of the two spatial objects are obvious in the 25IM. The corresponding topological part of $A$ is nonexistent when all the values of a row are empty, the corresponding topological part of $B$ is nonexistent when all the values of a column are empty.

- $\mathrm{A} / \mathrm{B}$ is a body if any value of the 4 th row/column is non-empty.

- $\mathrm{A} / \mathrm{B}$ is a region if all the values of the 4 th row/column are empty and any value of the 3rd row/column is non-empty.

- $\mathrm{A} / \mathrm{B}$ is a line if all the values of the $3 \mathrm{rd}$ and 4 th rows/columns are empty and any value of the 2 nd row/column is non-empty.

- $\mathrm{A} / \mathrm{B}$ is a point if all the values of the $2 \mathrm{nd}, 3 \mathrm{rd}$, and 4 th rows/columns are empty.

In order to simplify the description of topological relations, spatial object $A$ is always set to have a higher dimension than spatial object $B(\operatorname{dim}(A) \geq \operatorname{dim}(B))$. If $B$ has higher dimensions, then transpose the matrix. This paper does not differentiate cover/coveredby or inside/contain because " $\mathrm{A}$ is covered by $B$ " is equal to " $B$ covers $A$ " and " $A$ is inside $B$ " is equal to "B contains $A$ ". The matrix of " $A$ is covered by $B$ " is the transposed matrix of "A covers B", and the matrix of " $A$ is inside $B$ " is the transposed matrix of "A contains B". Thus, there are six kinds of topological relations between two spatial objects: disjoint, contain, equal, meet, cover, and overlap. Spatial object A is possible to be equal to spatial object B only when A and B have the same dimensions. Meet can be subdivided into meet at vertex, meet at edge, and meet at face. Cover can be subdivided into cover at vertex, cover at edge, and cover at face. There are a large number of topological relations that can be distinguished by the 25IM. Because some relations are similar, only the diagrams of some typical relations are shown in Figures 3 and 5, Figures 6-11.

\subsection{Body/Body Relations}

There are six kinds of body/body topological relations: disjoint, contain, equal, meet, cover, and overlap.

1. Disjoint (Figure 5a): A body/body disjoint relation means that body B locates in the exterior of body A. If the intersection of A's closure and B's closure is empty, then body A disjoints from body $\mathrm{B}$. There is only one body/body disjoint relation that can be realized based on the 25IM.

$$
\text { A.Disjoint }(\mathrm{B}) \rightarrow \overline{\mathrm{A}} \cap \overline{\mathrm{B}}=\varnothing
$$




$$
R_{25 I M}(A, B)=\left[\begin{array}{lllll}
0 & 0 & 0 & 0 & 1 \\
0 & 0 & 0 & 0 & 1 \\
0 & 0 & 0 & 0 & 1 \\
0 & 0 & 0 & 0 & 1 \\
1 & 1 & 1 & 1 & 1
\end{array}\right]
$$

2. Contain: A body/body contain relation means that body B is inside the interior of body A. If the intersection of A's interior and B's closure is equal to B's closure, then A contains B. There are two body/body contain relations that can be realized based on the 25IM: A contains B (Figure 5b) and B contains A (Figure 5c). No more details like this will be repeated below.

$$
\begin{gathered}
\text { A.Contain }(\mathrm{B}) \rightarrow\left(\mathrm{A}^{\mathrm{o}} \cap \overline{\mathrm{B}}=\overline{\mathrm{B}}\right) \\
R_{25 I M}(A, B)=\left[\begin{array}{lllll}
0 & 0 & 0 & 0 & 1 \\
0 & 0 & 0 & 0 & 1 \\
0 & 0 & 0 & 0 & 1 \\
1 & 1 & 1 & 1 & 1 \\
0 & 0 & 0 & 0 & 1
\end{array}\right] \vee\left[\begin{array}{lllll}
0 & 0 & 0 & 1 & 0 \\
0 & 0 & 0 & 1 & 0 \\
0 & 0 & 0 & 1 & 0 \\
0 & 0 & 0 & 1 & 0 \\
1 & 1 & 1 & 1 & 1
\end{array}\right],
\end{gathered}
$$

If we define the relation "inside", there will be two body/body inside relations: B is inside A (Figure $5 \mathrm{~b}$ ) and $\mathrm{A}$ is inside $\mathrm{B}$ (Figure $5 \mathrm{c}$ ), which have the same expressions as body/body contain relations. If we want to query "Is B inside A?", just change the query to "Does A contain B?". Thus, we merge the inside relation into the contain relation. For the same reason, we merge the coveredby relation into the cover relation.

3. Equal (Figure 5d): A body/body equal relation means that body A and body B are spatially the same. If a topological part of body A only intersects with the same topological part of body B, then $\mathrm{A}$ is equal to $\mathrm{B}$. There is only one body/body equal relation that can be realized based on the 25IM.

$$
\begin{gathered}
\text { A.Equal }(\mathrm{B}) \rightarrow\left(\mathrm{A}^{\mathrm{o}}=\mathrm{B}^{\mathrm{o}}\right) \wedge\left(\mathrm{A}^{\mathrm{v}}=\mathrm{B}^{\mathrm{v}}\right) \wedge\left(\mathrm{A}^{\mathrm{e}}=\mathrm{B}^{\mathrm{e}}\right) \wedge\left(\mathrm{A}^{\mathrm{f}}=\mathrm{B}^{\mathrm{f}}\right) \wedge\left(\mathrm{A}^{-}=\mathrm{B}^{-}\right) \\
R_{25 I M}(A, B)=\left[\begin{array}{lllll}
1 & 0 & 0 & 0 & 0 \\
0 & 1 & 0 & 0 & 0 \\
0 & 0 & 1 & 0 & 0 \\
0 & 0 & 0 & 1 & 0 \\
0 & 0 & 0 & 0 & 1
\end{array}\right]
\end{gathered}
$$

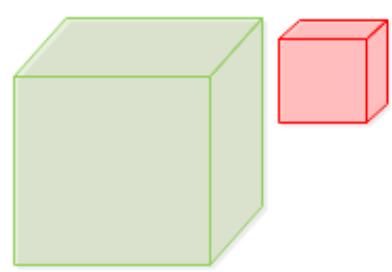

(a)

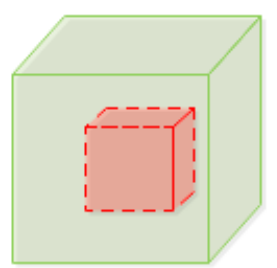

(b)

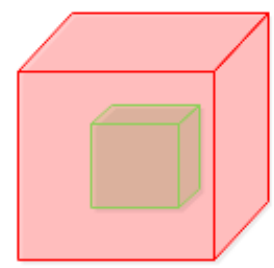

(c)

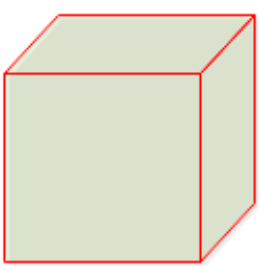

(d)

Figure 5. (a) Body/body disjoint, (b) body/body contain (A contains B), (c) body/body contain (B contains A), and (d) body/body equal.

4. Meet: A body/body meet relation means that body B touches body A at a vertex, edge, or face, and B's interior is located in A's exterior. If the intersection of A's interior and B's interior is 
empty, and the intersection of A's boundary and B's boundary is non-empty, then body A meets body B. There are three situations for the body/body meet relation.

$$
\operatorname{A.Meet}(\mathrm{B}) \rightarrow\left(\mathrm{A}^{\mathrm{o}} \cap \mathrm{B}^{\mathrm{o}}=\varnothing\right) \wedge\left(\mathrm{A}^{\mathrm{o}} \cap \partial \mathrm{B}=\varnothing\right) \wedge(\partial \mathrm{A} \cap \partial \mathrm{B} \neq \varnothing)
$$

(1) Meet at vertex (Figure 6): Body B's interior is inside body A's exterior. One or more of B's vertices touch A's vertex, edge, or face. However, no edge or face of B touches A's closure. There are $1+2+4+4+2=13$ body/body meet-at-vertex relations that can be realized based on the 25IM.

$$
\begin{aligned}
& \left(\partial \mathrm{A} \cap \mathrm{B}^{\mathrm{v}} \neq \varnothing\right) \wedge\left(\partial \mathrm{A} \cap \mathrm{B}^{\mathrm{e}}=\varnothing\right) \wedge\left(\partial \mathrm{A} \cap \mathrm{B}^{\mathrm{f}}=\varnothing\right) \\
R_{25 I M}(A, B)= & {\left[\begin{array}{lllll}
1 & 0 & 0 & 0 & 1 \\
0 & 0 & 0 & 0 & 1 \\
0 & 0 & 0 & 0 & 1 \\
0 & 0 & 0 & 0 & 1 \\
1 & 1 & 1 & 1 & 1
\end{array}\right] \vee\left[\begin{array}{ccccc}
* & 0 & 0 & 0 & 1 \\
1 & 0 & 0 & 0 & 1 \\
0 & 0 & 0 & 0 & 1 \\
0 & 0 & 0 & 0 & 1 \\
1 & 1 & 1 & 1 & 1
\end{array}\right] \vee\left[\begin{array}{lllll}
* & 0 & 0 & 0 & 1 \\
* & 0 & 0 & 0 & 1 \\
1 & 0 & 0 & 0 & 1 \\
0 & 0 & 0 & 0 & 1 \\
1 & 1 & 1 & 1 & 1
\end{array}\right] } \\
& \vee\left[\begin{array}{ccccc}
* & * & 1 & 0 & 1 \\
0 & 0 & 0 & 0 & 1 \\
0 & 0 & 0 & 0 & 1 \\
0 & 0 & 0 & 0 & 1 \\
1 & 1 & 1 & 1 & 1
\end{array}\right] \vee\left[\begin{array}{ccccc}
* & 1 & 0 & 0 & 1 \\
0 & 0 & 0 & 0 & 1 \\
0 & 0 & 0 & 0 & 1 \\
0 & 0 & 0 & 0 & 1 \\
1 & 1 & 1 & 1 & 1
\end{array}\right],
\end{aligned}
$$

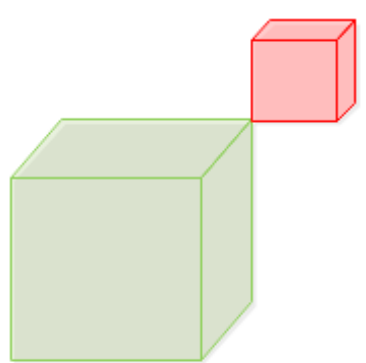

(a)

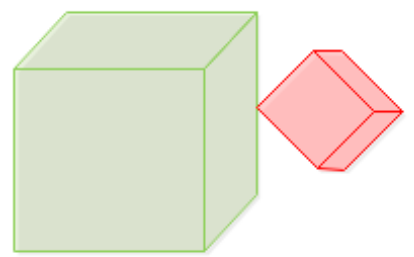

(b)

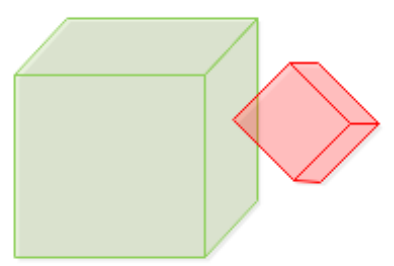

(c)

Figure 6. Body/body meet at vertex: (a) A and B share a common vertex, (b) A's edge contains B's vertex, (c) A's face contains B's vertex.

(2) Meet at edge (Figure 7): Body B's interior is inside body A's exterior. One or more of B's edges touches $A^{\prime}$ s edges or faces. A and B may share common vertices, but no face of B touches A's face. There are $2^{3}+2^{7}+2^{6}=200$ body/body meet-at-edge relations that can be realized based on the 25IM.

$$
\begin{gathered}
\left(\left(\mathrm{A}^{\mathrm{e}}+\mathrm{A}^{\mathrm{f}}\right) \cap \mathrm{B}^{\mathrm{e}} \neq \varnothing\right) \wedge\left(\mathrm{A}^{\mathrm{f}} \cap \mathrm{B}^{\mathrm{f}}=\varnothing\right) \\
R_{25 I M}(A, B)=\left[\begin{array}{ccccc}
* & * & 0 & 0 & 1 \\
* & 1 & 0 & 0 & 1 \\
0 & 0 & 0 & 0 & 1 \\
0 & 0 & 0 & 0 & 1 \\
1 & 1 & 1 & 1 & 1
\end{array}\right] \vee\left[\begin{array}{ccccc}
* & * & * & 0 & 1 \\
* & * & * & 0 & 1 \\
* & 1 & 0 & 0 & 1 \\
0 & 0 & 0 & 0 & 1 \\
1 & 1 & 1 & 1 & 1
\end{array}\right] \vee\left[\begin{array}{ccccc}
* & * & * & 0 & 1 \\
* & * & 1 & 0 & 1 \\
* & 0 & 0 & 0 & 1 \\
0 & 0 & 0 & 0 & 1 \\
1 & 1 & 1 & 1 & 1
\end{array}\right],
\end{gathered}
$$

(3) Meet at face (Figure 3): Body B's interior is inside body A's exterior. B's face touches A's face. A and B may share common vertices or edges. There are $2^{8}-2^{3}=248$ body/body meet-at-face relations that can be realized based on the 25IM. 


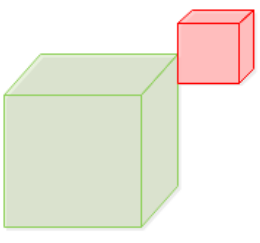

(a)

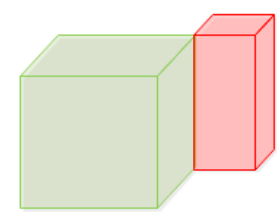

(b)

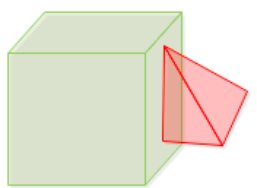

(c)

Figure 7. Body/body meet at edge: (a) A's edge overlaps B's edge, (b) A's edge is equal to B's edge, (c) A's face contains B's edge.

Theorem 2. If body A meets body $B$ at a face, there must be either a B's vertex on A's boundary or an A's vertex on B's boundary.

$$
\begin{gathered}
\left(A^{f} \cap B^{f} \neq \varnothing\right) \wedge\left(\partial A \cap B^{v} \neq \varnothing\right) \\
R_{25 I M}(A, B)=\left[\begin{array}{ccccc}
* & * & * & 0 & 1 \\
* & * & * & 0 & 1 \\
* & * & 1 & 0 & 1 \\
0 & 0 & 0 & 0 & 1 \\
1 & 1 & 1 & 1 & 1
\end{array}\right], \\
R_{25 I M}(A, B) \neq\left[\begin{array}{lllll}
0 & 0 & 0 & 0 & 1 \\
0 & * & * & 0 & 1 \\
0 & * & 1 & 0 & 1 \\
0 & 0 & 0 & 0 & 1 \\
1 & 1 & 1 & 1 & 1
\end{array}\right],
\end{gathered}
$$

Therefore, there are $13+200+248=461$ body/body meet relations that can be realized based on the 25IM.

5. Cover: A body/body cover relation means that body B's interior is inside body A's closure and body B touches body A at a vertex, edge, or face. If the intersection of A's closure and B's closure is equal to B's closure, the intersection of A's exterior and B's closure is empty, the intersection of A's interior and B's boundary is non-empty, and the intersection of A's boundary and B's boundary is non-empty, then A covers B. There are three situations for the body/body cover relation.

Theorem 3. If body A cover body B, there must be a B's vertex on A's boundary.

$$
\begin{gathered}
\text { A.Cover }(\mathrm{B}) \rightarrow(\overline{\mathrm{A}} \cap \overline{\mathrm{B}}=\overline{\mathrm{B}}) \wedge\left(\mathrm{A}^{-} \cap \overline{\mathrm{B}}=\varnothing\right) \wedge\left(\mathrm{A}^{\mathrm{o}} \cap \partial \mathrm{B} \neq \varnothing\right) \wedge(\partial \mathrm{A} \cap \partial \mathrm{B} \neq \varnothing) \\
\wedge\left(\partial \mathrm{A} \cap \mathrm{B}^{\vee} \neq \varnothing\right)
\end{gathered}
$$

(1) Cover at vertex (Figure 8): Body B's interior is inside body A's closure. One or more of B's vertices touches A's vertex, edge, or face. However, no edge or face of B touches A's boundary. There are $(1+2+4) \times 2=14$ body/body cover-at-vertex relations that can be realized based on the 25IM.

$$
\left(\partial \mathrm{A} \cap \mathrm{B}^{\mathrm{v}} \neq \varnothing\right) \wedge\left(\partial \mathrm{A} \cap \mathrm{B}^{\mathrm{e}}=\varnothing\right) \wedge\left(\partial \mathrm{A} \cap \mathrm{B}^{\mathrm{f}}=\varnothing\right)
$$

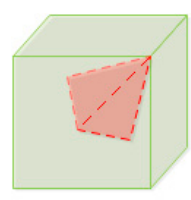

(a)

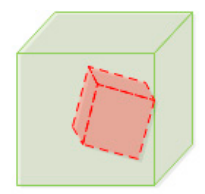

(b)

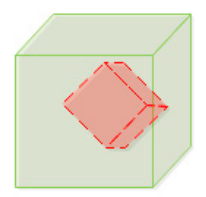

(c)

Figure 8. Body/body cover at vertex: (a) A and B share a common vertex, (b) A's edge contains B's vertex, (c) A's face contains B's vertex. 


$$
\begin{aligned}
R_{25 I M}(A, B) & =\left[\begin{array}{lllll}
1 & 0 & 0 & 0 & 1 \\
0 & 0 & 0 & 0 & 1 \\
0 & 0 & 0 & 0 & 1 \\
1 & 1 & 1 & 1 & 1 \\
0 & 0 & 0 & 0 & 1
\end{array}\right] \vee\left[\begin{array}{lllll}
* & 0 & 0 & 0 & 1 \\
1 & 0 & 0 & 0 & 1 \\
0 & 0 & 0 & 0 & 1 \\
1 & 1 & 1 & 1 & 1 \\
0 & 0 & 0 & 0 & 1
\end{array}\right] \vee\left[\begin{array}{ccccc}
* & 0 & 0 & 0 & 1 \\
* & 0 & 0 & 0 & 1 \\
1 & 0 & 0 & 0 & 1 \\
1 & 1 & 1 & 1 & 1 \\
0 & 0 & 0 & 0 & 1
\end{array}\right] \\
& \vee\left[\begin{array}{lllll}
1 & 0 & 0 & 1 & 0 \\
0 & 0 & 0 & 1 & 0 \\
0 & 0 & 0 & 1 & 0 \\
0 & 0 & 0 & 1 & 0 \\
1 & 1 & 1 & 1 & 1
\end{array}\right] \vee\left[\begin{array}{lllll}
* & 1 & 0 & 1 & 0 \\
0 & 0 & 0 & 1 & 0 \\
0 & 0 & 0 & 1 & 0 \\
0 & 0 & 0 & 1 & 0 \\
1 & 1 & 1 & 1 & 1
\end{array}\right] \vee\left[\begin{array}{lllll}
* & * & 1 & 0 \\
0 & 0 & 0 & 1 & 0 \\
0 & 0 & 0 & 1 & 0 \\
0 & 0 & 0 & 1 & 0 \\
1 & 1 & 1 & 1 & 1
\end{array}\right],
\end{aligned}
$$

(2) Cover at edge (Figure 9): Body B's interior is inside body A's closure. One or more B's edges touch body A's edge or face. A and B may share common vertices, but no face of B touches A's boundary. There are $\left(2^{3}+2^{4}-2-2\right) \times 2=40$ body/body cover-at-edge relations that can be realized based on the 25IM.

$$
\begin{aligned}
& \left(\left(\mathrm{A}^{\mathrm{e}}+\mathrm{A}^{\mathrm{f}}\right) \cap \mathrm{B}^{\mathrm{e}} \neq \varnothing\right) \wedge\left(\partial \mathrm{A} \cap \mathrm{B}^{\mathrm{f}}=\varnothing\right) \wedge\left(\left(\mathrm{A}^{\mathrm{v}}+\mathrm{A}^{\mathrm{e}}\right) \cap \mathrm{B}^{\mathrm{v}} \neq \varnothing\right) \\
& R_{25 I M}(A, B)=\left[\begin{array}{lllll}
* & 0 & 0 & 0 & 1 \\
* & 1 & 0 & 0 & 1 \\
* & 0 & 0 & 0 & 1 \\
1 & 1 & 1 & 1 & 1 \\
0 & 0 & 0 & 0 & 1
\end{array}\right] \vee\left[\begin{array}{ccccc}
* & 0 & 0 & 0 & 1 \\
* & * & 0 & 0 & 1 \\
* & 1 & 0 & 0 & 1 \\
1 & 1 & 1 & 1 & 1 \\
0 & 0 & 0 & 0 & 1
\end{array}\right] \vee\left[\begin{array}{ccccc}
* & * & * & 1 & 0 \\
0 & 1 & 0 & 1 & 0 \\
0 & 0 & 0 & 1 & 0 \\
0 & 0 & 0 & 1 & 0 \\
1 & 1 & 1 & 1 & 1
\end{array}\right] \vee\left[\begin{array}{ccccc}
* & * & * & 1 & 0 \\
0 & * & 1 & 1 & 0 \\
0 & 0 & 0 & 1 & 0 \\
0 & 0 & 0 & 1 & 0 \\
1 & 1 & 1 & 1 & 1
\end{array}\right], \\
& R_{25 I M}(A, B) \neq\left[\begin{array}{lllll}
0 & 0 & 0 & 0 & 1 \\
0 & 1 & 0 & 0 & 1 \\
* & 0 & 0 & 0 & 1 \\
1 & 1 & 1 & 1 & 1 \\
0 & 0 & 0 & 0 & 1
\end{array}\right] \vee\left[\begin{array}{lllll}
0 & 0 & 0 & 0 & 1 \\
0 & * & 0 & 0 & 1 \\
0 & 1 & 0 & 0 & 1 \\
1 & 1 & 1 & 1 & 1 \\
0 & 0 & 0 & 0 & 1
\end{array}\right] \vee\left[\begin{array}{lllll}
0 & 0 & * & 1 & 0 \\
0 & 1 & 0 & 1 & 0 \\
0 & 0 & 0 & 1 & 0 \\
0 & 0 & 0 & 1 & 0 \\
1 & 1 & 1 & 1 & 1
\end{array}\right] \vee\left[\begin{array}{ccccc}
0 & 0 & 0 & 1 & 0 \\
0 & * & 1 & 1 & 0 \\
0 & 0 & 0 & 1 & 0 \\
0 & 0 & 0 & 1 & 0 \\
1 & 1 & 1 & 1 & 1
\end{array}\right],
\end{aligned}
$$
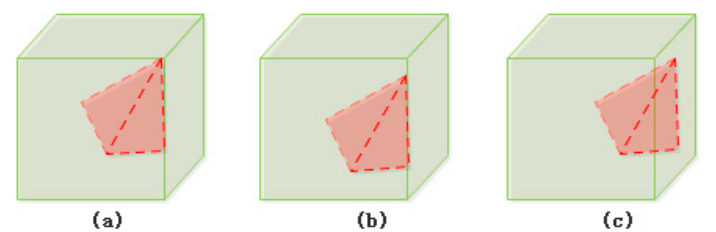

Figure 9. Body/body cover at edge: (a) A's edge covers B's edge, (b) A's edge contains B's edge, (c) A's face contains B's edge.

(3) Cover at face (Figure 10): Body B's interior is inside body A's closure. B's face touches A's faces. $A$ and $B$ may share common vertex or edge. There are $\left(2^{6}-2^{3}+2^{5}-2^{2}\right) \times 2=84$ body/body cover-at-face relations that can be realized based on the 25IM.

$$
A^{f} \cap B^{f} \neq \varnothing
$$
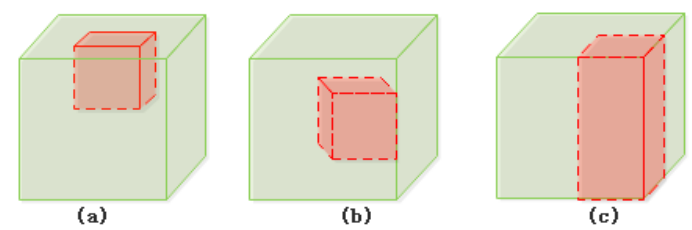

Figure 10. Body/body cover at face: (a) A's face contains B's face, (b) A's face covers B's face, (c) A and $B$ share a common edge. 


$$
\begin{aligned}
& R_{25 I M}(A, B)=\left[\begin{array}{lllll}
* & 0 & 0 & 0 & 1 \\
* & * & 0 & 0 & 1 \\
* & * & 1 & 0 & 1 \\
0 & * & 1 & 1 & 1 \\
0 & 0 & 0 & 0 & 1
\end{array}\right] \vee\left[\begin{array}{lllll}
* & 0 & 0 & 0 & 1 \\
* & * & 0 & 0 & 1 \\
* & * & 1 & 0 & 1 \\
1 & 1 & 1 & 1 & 1 \\
0 & 0 & 0 & 0 & 1
\end{array}\right] \vee\left[\begin{array}{ccccc}
* & * & * & 1 & 0 \\
0 & * & * & 1 & 0 \\
0 & 0 & 1 & 1 & 0 \\
0 & 0 & 0 & 1 & 0 \\
1 & 1 & 1 & 1 & 1
\end{array}\right] \vee\left[\begin{array}{ccccc}
* & * & * & 0 & 0 \\
0 & * & * & * & 0 \\
0 & 0 & 1 & 1 & 0 \\
0 & 0 & 0 & 1 & 0 \\
1 & 1 & 1 & 1 & 1
\end{array}\right], \\
& R_{25 I M}(A, B) \neq\left[\begin{array}{lllll}
0 & 0 & 0 & 0 & 1 \\
0 & * & 0 & 0 & 1 \\
0 & * & 1 & 0 & 1 \\
0 & * & 1 & 1 & 1 \\
0 & 0 & 0 & 0 & 1
\end{array}\right] \vee\left[\begin{array}{lllll}
0 & 0 & 0 & 0 & 1 \\
0 & * & 0 & 0 & 1 \\
0 & * & 1 & 0 & 1 \\
1 & 1 & 1 & 1 & 1 \\
0 & 0 & 0 & 0 & 1
\end{array}\right] \vee\left[\begin{array}{lllll}
0 & 0 & 0 & 1 & 0 \\
0 & * & * & 1 & 0 \\
0 & 0 & 1 & 1 & 0 \\
0 & 0 & 0 & 1 & 0 \\
1 & 1 & 1 & 1 & 1
\end{array}\right] \vee\left[\begin{array}{lllll}
0 & 0 & 0 & 0 & 0 \\
0 & * & * & * & 0 \\
0 & 0 & 1 & 1 & 0 \\
0 & 0 & 0 & 1 & 0 \\
1 & 1 & 1 & 1 & 1
\end{array}\right],
\end{aligned}
$$

Therefore, there are $14+40+84=138$ body/body cover relations that can be realized based on the 25IM.

6. Overlap (Figure 11): A body/body overlap relation means that body A's interior intersects with body B's interior, but A does not contain, does not cover, or is not equal to B. If the intersection of A's interior and B's interior is non-empty, the intersection of A's interior and B's boundary is non-empty, and the intersection of A's boundary and B's boundary is non-empty, then A overlaps B.

$$
\begin{gathered}
\text { A.Overlap }(\mathrm{B}) \rightarrow\left(\mathrm{A}^{\mathrm{o}} \cap \mathrm{B}^{\mathrm{o}} \neq \varnothing\right) \wedge\left(\mathrm{A}^{\mathrm{o}} \cap \partial \mathrm{B} \neq \varnothing\right) \wedge(\partial \mathrm{A} \cap \partial \mathrm{B} \neq \varnothing) \\
\qquad R_{25 I M}(A, B)=\left[\begin{array}{ccccc}
* & * & * & * & 1 \\
* & * & * & * & 1 \\
* & * & 1 & 1 & 1 \\
* & * & 1 & 1 & 1 \\
1 & 1 & 1 & 1 & 1
\end{array}\right]
\end{gathered}
$$

Theorem 4. If a vertex of body $B$ intersects with the interior of body $A$, then there must be an edge and $a$ face of $B$ that intersect with the interior of $A$. Thus, if $A^{o} \cap B^{v} \neq \varnothing$, then $A^{o} \cap B^{e} \neq \varnothing$.

$$
R_{25 I M}(A, B) \neq\left[\begin{array}{lllll}
* & * & * & 1 & 1 \\
* & * & * & 0 & 1 \\
* & * & 1 & 1 & 1 \\
* & * & 1 & 1 & 1 \\
1 & 1 & 1 & 1 & 1
\end{array}\right] \vee\left[\begin{array}{ccccc}
* & * & * & * & 1 \\
* & * & * & * & 1 \\
* & * & 1 & 1 & 1 \\
1 & 0 & 1 & 1 & 1 \\
1 & 1 & 1 & 1 & 1
\end{array}\right],
$$

There are $2^{12}-2^{10}-2^{10}=2048$ body/body overlap relations that can be realized based on the 25IM.

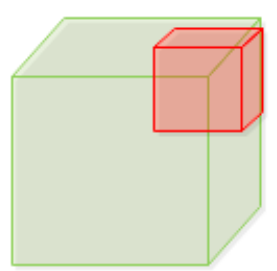

(a)

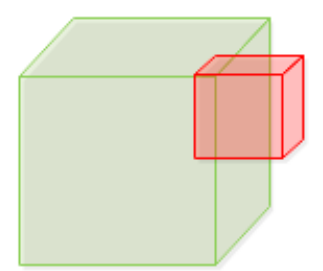

(b)

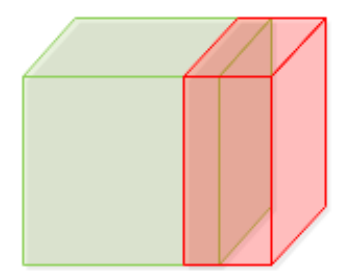

(c)

Figure 11. Body/body overlap: (a) A's edges are disjoint from B's edges, (b) A's face covers B's edge at B's vertex, (c) A's face covers B's edge at B's edge. 
Therefore, there are $1+2+1+461+138+2048=2651$ body/body relations that can be realized based on the 25IM.

The following relations are dimensionally reduced situations of body/body relations. Therefore, we only give their expressions without detailed descriptions.

\subsection{Body/Region Relations}

There are five kinds of body/region topological relations: disjoint, contain, meet, cover, and overlap. According to the definition above, region B has vertex, edge, face, and exterior but no interior. Therefore, the intersections of all A's topological part and B's interior are empty.

1. Disjoint: There are two body/region disjoint relations that can be realized based on the 25IM.

$$
R_{25 I M}(A, B)=\left[\begin{array}{lllll}
0 & 0 & 0 & 0 & 1 \\
0 & 0 & 0 & 0 & 1 \\
0 & 0 & 0 & 0 & 1 \\
0 & 0 & 0 & 0 & 1 \\
1 & 1 & 1 & 0 & 1
\end{array}\right] \vee\left[\begin{array}{lllll}
0 & 0 & 0 & 0 & 1 \\
0 & 0 & 0 & 0 & 1 \\
0 & 0 & 0 & 0 & 1 \\
0 & 0 & 0 & 0 & 0 \\
1 & 1 & 1 & 1 & 1
\end{array}\right]
$$

2. Contain: There are two body/region contain relations that can be realized based on the 25IM.

$$
R_{25 I M}(A, B)=\left[\begin{array}{lllll}
0 & 0 & 0 & 0 & 1 \\
0 & 0 & 0 & 0 & 1 \\
0 & 0 & 0 & 0 & 1 \\
1 & 1 & 1 & 0 & 1 \\
0 & 0 & 0 & 0 & 1
\end{array}\right] \vee\left[\begin{array}{lllll}
0 & 0 & 0 & 1 & 0 \\
0 & 0 & 0 & 1 & 0 \\
0 & 0 & 0 & 1 & 0 \\
0 & 0 & 0 & 0 & 0 \\
1 & 1 & 1 & 1 & 1
\end{array}\right]
$$

3. Meet:

(1) Meet at vertex: There are $(1+2+4) \times 2=14$ body/region meet-at-vertex relations that can be realized based on the 25IM.

$$
\begin{aligned}
R_{25 I M}(A, B) & =\left[\begin{array}{lllll}
1 & 0 & 0 & 0 & 1 \\
0 & 0 & 0 & 0 & 1 \\
0 & 0 & 0 & 0 & 1 \\
0 & 0 & 0 & 0 & 1 \\
1 & 1 & 1 & 0 & 1
\end{array}\right] \vee\left[\begin{array}{ccccc}
* & 0 & 0 & 0 & 1 \\
1 & 0 & 0 & 0 & 1 \\
0 & 0 & 0 & 0 & 1 \\
0 & 0 & 0 & 0 & 1 \\
1 & 1 & 1 & 0 & 1
\end{array}\right] \vee\left[\begin{array}{ccccc}
* & 0 & 0 & 0 & 1 \\
* & 0 & 0 & 0 & 1 \\
1 & 0 & 0 & 0 & 1 \\
0 & 0 & 0 & 0 & 1 \\
1 & 1 & 1 & 0 & 1
\end{array}\right] \\
& \vee\left[\begin{array}{ccccc}
* & * & 1 & 0 & 1 \\
0 & 0 & 0 & 0 & 1 \\
0 & 0 & 0 & 0 & 1 \\
0 & 0 & 0 & 0 & 0 \\
1 & 1 & 1 & 1 & 1
\end{array}\right] \vee\left[\begin{array}{lllll}
* & 1 & 0 & 0 & 1 \\
0 & 0 & 0 & 0 & 1 \\
0 & 0 & 0 & 0 & 1 \\
0 & 0 & 0 & 0 & 0 \\
1 & 1 & 1 & 1 & 1
\end{array}\right] \vee\left[\begin{array}{lllll}
1 & 0 & 0 & 0 & 1 \\
0 & 0 & 0 & 0 & 1 \\
0 & 0 & 0 & 0 & 1 \\
0 & 0 & 0 & 0 & 0 \\
1 & 1 & 1 & 1 & 1
\end{array}\right],
\end{aligned}
$$

(2) Meet at edge: There are $\left(2^{3}+2^{7}+2^{6}\right) \times 2=400$ body/region meet-at-edge relations that can be realized based on the 25IM.

$$
\begin{aligned}
R_{25 I M}(A, B) & =\left[\begin{array}{lllll}
* & * & 0 & 0 & 1 \\
* & 1 & 0 & 0 & 1 \\
0 & 0 & 0 & 0 & 1 \\
0 & 0 & 0 & 0 & 1 \\
1 & 1 & 1 & 0 & 1
\end{array}\right] \vee\left[\begin{array}{lllll}
* & * & * & 0 & 1 \\
* & * & * & 0 & 1 \\
* & 1 & 0 & 0 & 1 \\
0 & 0 & 0 & 0 & 1 \\
1 & 1 & 1 & 0 & 1
\end{array}\right] \vee\left[\begin{array}{ccccc}
* & * & * & 0 & 1 \\
* & * & 1 & 0 & 1 \\
* & 0 & 0 & 0 & 1 \\
0 & 0 & 0 & 0 & 1 \\
1 & 1 & 1 & 0 & 1
\end{array}\right] \\
& \vee\left[\begin{array}{ccccc}
* & * & 0 & 0 & 1 \\
* & 1 & 0 & 0 & 1 \\
0 & 0 & 0 & 0 & 1 \\
0 & 0 & 0 & 0 & 0 \\
1 & 1 & 1 & 1 & 1
\end{array}\right] \vee\left[\begin{array}{lllll}
* & * & * & 0 & 1 \\
* & * & 1 & 0 & 1 \\
* & * & 0 & 0 & 1 \\
0 & 0 & 0 & 0 & 0 \\
1 & 1 & 1 & 1 & 1
\end{array}\right] \vee\left[\begin{array}{lllll}
* & * & 0 & 1 \\
* & * & 0 & 0 & 1 \\
* & 1 & 0 & 0 & 1 \\
0 & 0 & 0 & 0 & 0 \\
1 & 1 & 1 & 1 & 1
\end{array}\right],
\end{aligned}
$$


(3) Meet at face: There are $\left(2^{8}-2^{3}\right) \times 2=496$ body/region meet-at-face relations that can be realized based on the 25IM.

$$
\begin{aligned}
& R_{25 I M}(A, B)=\left[\begin{array}{lllll}
* & * & * & 0 & 1 \\
* & * & * & 0 & 1 \\
* & * & 1 & 0 & 1 \\
0 & 0 & 0 & 0 & 1 \\
1 & 1 & 1 & 0 & 1
\end{array}\right] \vee\left[\begin{array}{lllll}
* & * & * & 0 & 1 \\
* & * & * & 0 & 1 \\
* & * & 1 & 0 & 1 \\
0 & 0 & 0 & 0 & 0 \\
1 & 1 & 1 & 1 & 1
\end{array}\right], \\
& R_{25 I M}(A, B) \neq\left[\begin{array}{lllll}
0 & 0 & 0 & 0 & 1 \\
0 & * & * & 0 & 1 \\
0 & * & 1 & 0 & 1 \\
0 & 0 & 0 & 0 & 1 \\
1 & 1 & 1 & 0 & 1
\end{array}\right] \vee\left[\begin{array}{lllll}
0 & 0 & 0 & 0 & 1 \\
0 & * & * & 0 & 1 \\
0 & * & 1 & 0 & 1 \\
0 & 0 & 0 & 0 & 0 \\
1 & 1 & 1 & 1 & 1
\end{array}\right],
\end{aligned}
$$

Therefore, there are $14+400+496=910$ body/region meet relations that can be realized based on the 25IM.

\section{Cover:}

(1) Cover at vertex: There are $(1+2+4) \times 2=14$ body/region cover-at-vertex relations that can be realized based on the 25IM.

$$
\begin{gathered}
R_{25 I M}(A, B)=\left[\begin{array}{lllll}
1 & 0 & 0 & 0 & 1 \\
0 & 0 & 0 & 0 & 1 \\
0 & 0 & 0 & 0 & 1 \\
1 & 1 & 1 & 0 & 1 \\
0 & 0 & 0 & 0 & 1
\end{array}\right] \vee\left[\begin{array}{lllll}
* & 0 & 0 & 0 & 1 \\
1 & 0 & 0 & 0 & 1 \\
0 & 0 & 0 & 0 & 1 \\
1 & 1 & 1 & 0 & 1 \\
0 & 0 & 0 & 0 & 1
\end{array}\right] \vee\left[\begin{array}{lllll}
* & 0 & 0 & 0 & 1 \\
* & 0 & 0 & 0 & 1 \\
1 & 0 & 0 & 0 & 1 \\
1 & 1 & 1 & 0 & 1 \\
0 & 0 & 0 & 0 & 1
\end{array}\right] \\
\vee\left[\begin{array}{lllll}
1 & 0 & 0 & 1 & 0 \\
0 & 0 & 0 & 1 & 0 \\
0 & 0 & 0 & 1 & 0 \\
0 & 0 & 0 & 0 & 0 \\
1 & 1 & 1 & 1 & 1
\end{array}\right] \vee\left[\begin{array}{lllll}
* & 1 & 0 & 1 & 0 \\
0 & 0 & 0 & 1 & 0 \\
0 & 0 & 0 & 1 & 0 \\
0 & 0 & 0 & 0 & 0 \\
1 & 1 & 1 & 1 & 1
\end{array}\right] \vee\left[\begin{array}{lllll}
* & 1 & 1 & 0 \\
0 & 0 & 0 & 1 & 0 \\
0 & 0 & 0 & 1 & 0 \\
0 & 0 & 0 & 0 & 0 \\
1 & 1 & 1 & 1 & 1
\end{array}\right]
\end{gathered}
$$

(2) Cover at edge: There are $\left(2^{3}+2^{4}-2-2\right) \times 2=40$ body/region cover-at-edge relations that can be realized based on the 25IM.

$$
\begin{aligned}
& R_{25 I M}(A, B)=\left[\begin{array}{ccccc}
* & 0 & 0 & 0 & 1 \\
* & 1 & 0 & 0 & 1 \\
* & 0 & 0 & 0 & 1 \\
1 & 1 & 1 & 0 & 1 \\
0 & 0 & 0 & 0 & 1
\end{array}\right] \vee\left[\begin{array}{ccccc}
* & 0 & 0 & 0 & 1 \\
* & * & 0 & 0 & 1 \\
* & 1 & 0 & 0 & 1 \\
1 & 1 & 1 & 0 & 1 \\
0 & 0 & 0 & 0 & 1
\end{array}\right] \vee\left[\begin{array}{ccccc}
* & * & * & 1 & 0 \\
0 & 1 & 0 & 1 & 0 \\
0 & 0 & 0 & 1 & 0 \\
0 & 0 & 0 & 0 & 0 \\
1 & 1 & 1 & 1 & 1
\end{array}\right] \vee\left[\begin{array}{ccccc}
* & * & * & 1 & 0 \\
0 & * & 1 & 1 & 0 \\
0 & 0 & 0 & 1 & 0 \\
0 & 0 & 0 & 0 & 0 \\
1 & 1 & 1 & 1 & 1
\end{array}\right], \\
& R_{25 I M}(A, B) \neq\left[\begin{array}{lllll}
0 & 0 & 0 & 0 & 1 \\
0 & 1 & 0 & 0 & 1 \\
* & 0 & 0 & 0 & 1 \\
1 & 1 & 1 & 0 & 1 \\
0 & 0 & 0 & 0 & 1
\end{array}\right] \vee\left[\begin{array}{lllll}
0 & 0 & 0 & 0 & 1 \\
0 & * & 0 & 0 & 1 \\
0 & 1 & 0 & 0 & 1 \\
1 & 1 & 1 & 0 & 1 \\
0 & 0 & 0 & 0 & 1
\end{array}\right] \vee\left[\begin{array}{lllll}
0 & 0 & * & 1 & 0 \\
0 & 1 & 0 & 1 & 0 \\
0 & 0 & 0 & 1 & 0 \\
0 & 0 & 0 & 0 & 0 \\
1 & 1 & 1 & 1 & 1
\end{array}\right] \vee\left[\begin{array}{lllll}
0 & 0 & 0 & 1 & 0 \\
0 & * & 1 & 1 & 0 \\
0 & 0 & 0 & 1 & 0 \\
0 & 0 & 0 & 0 & 0 \\
1 & 1 & 1 & 1 & 1
\end{array}\right],
\end{aligned}
$$


(3) Cover at face: There are $\left(2^{6}-2^{3}+2^{5}-2^{2}\right) \times 2=84$ body/region cover-at-face relations that can be realized based on the 25IM.

$$
\begin{aligned}
& R_{25 I M}(A, B)=\left[\begin{array}{ccccc}
* & 0 & 0 & 0 & 1 \\
* & * & 0 & 0 & 1 \\
* & * & 1 & 0 & 1 \\
0 & * & 1 & 0 & 1 \\
0 & 0 & 0 & 0 & 1
\end{array}\right] \vee\left[\begin{array}{ccccc}
* & 0 & 0 & 0 & 1 \\
* & * & 0 & 0 & 1 \\
* & * & 1 & 0 & 1 \\
1 & 1 & 1 & 0 & 1 \\
0 & 0 & 0 & 0 & 1
\end{array}\right] \vee\left[\begin{array}{lllll}
* & * & * & 1 & 0 \\
0 & * & * & 1 & 0 \\
0 & 0 & 1 & 1 & 0 \\
0 & 0 & 0 & 0 & 0 \\
1 & 1 & 1 & 1 & 1
\end{array}\right] \vee\left[\begin{array}{ccccc}
* & * & * & 0 & 0 \\
0 & * & * & * & 0 \\
0 & 0 & 1 & 1 & 0 \\
0 & 0 & 0 & 0 & 0 \\
1 & 1 & 1 & 1 & 1
\end{array}\right], \\
& R_{25 I M}(A, B) \neq\left[\begin{array}{lllll}
0 & 0 & 0 & 0 & 1 \\
0 & * & 0 & 0 & 1 \\
0 & * & 1 & 0 & 1 \\
0 & 0 & 1 & 0 & 1 \\
0 & 0 & 1
\end{array}\right] \vee\left[\begin{array}{lllll}
0 & 0 & 0 & 0 & 1 \\
0 & * & 0 & 0 & 1 \\
0 & * & 1 & 0 & 1 \\
1 & 1 & 1 & 0 & 1 \\
0 & 0 & 0 & 0 & 1
\end{array}\right] \vee\left[\begin{array}{lllll}
0 & 0 & 0 & 1 & 0 \\
0 & * & * & 1 & 0 \\
0 & 0 & 1 & 1 & 0 \\
0 & 0 & 0 & 0 & 0 \\
1 & 1 & 1 & 1 & 1
\end{array}\right] \vee\left[\begin{array}{lllll}
0 & 0 & 0 & 0 & 0 \\
0 & * & * & * & 0 \\
0 & 0 & 1 & 1 & 0 \\
0 & 0 & 0 & 0 & 0 \\
1 & 1 & 1 & 1 & 1
\end{array}\right],
\end{aligned}
$$

Therefore, there are $14+40+84=138$ body/region cover relations that can be realized based on the 25IM.

5. Overlap: There are $\left(2^{10}-2^{8}\right) \times 2=1536$ body/region cover relations that can be realized based on the 25IM.

$$
R_{25 I M}(A, B)=\left[\begin{array}{lllll}
* & * & * & 0 & 1 \\
* & * & * & 0 & 1 \\
* & * & 1 & 0 & 1 \\
* & * & 1 & 0 & 1 \\
1 & 1 & 1 & 0 & 1
\end{array}\right] \vee\left[\begin{array}{lllll}
* & * & * & * & 1 \\
* & * & * & * & 1 \\
* & * & 1 & 1 & 1 \\
0 & 0 & 0 & 0 & 0 \\
1 & 1 & 1 & 1 & 1
\end{array}\right],
$$

Theorem 5. If a vertex of face $B$ intersects with the interior of body $A$, then there must be an edge of $B$ intersects with the interior of $A$. Thus, if $A^{o} \cap B^{v} \neq \varnothing$, then $A^{o} \cap B^{e} \neq \varnothing$.

$$
R_{25 I M}(A, B) \neq\left[\begin{array}{lllll}
* & * & * & 0 & 1 \\
* & * & * & 0 & 1 \\
* & * & 1 & 0 & 1 \\
1 & 0 & 1 & 0 & 1 \\
1 & 1 & 1 & 0 & 1
\end{array}\right] \vee\left[\begin{array}{lllll}
* & * & * & 1 & 1 \\
* & * & * & 0 & 1 \\
* & * & 1 & 1 & 1 \\
0 & 0 & 0 & 0 & 0 \\
1 & 1 & 1 & 1 & 1
\end{array}\right]
$$

Therefore, there are $2+2+910+138+1536=2588$ body/region relations that can be realized based on the 25IM.

\subsection{Body/Line Relations}

There are five kinds of body/region topological relations: disjoint, contain, meet, cover, and overlap. According to the definition above, line B only has vertex, edge, and exterior but no face and interior. Therefore, the intersections of all A's topological part and B's face/interior are empty.

1. Disjoint: There are two body/line disjoint relations that can be realized based on the 25IM.

$$
R_{25 I M}(A, B)=\left[\begin{array}{lllll}
0 & 0 & 0 & 0 & 1 \\
0 & 0 & 0 & 0 & 1 \\
0 & 0 & 0 & 0 & 1 \\
0 & 0 & 0 & 0 & 1 \\
1 & 1 & 0 & 0 & 1
\end{array}\right] \vee\left[\begin{array}{lllll}
0 & 0 & 0 & 0 & 1 \\
0 & 0 & 0 & 0 & 1 \\
0 & 0 & 0 & 0 & 0 \\
0 & 0 & 0 & 0 & 0 \\
1 & 1 & 1 & 1 & 1
\end{array}\right]
$$


2. Contain: There are two body/line contain relations that can be realized based on the 25IM.

$$
R_{25 I M}(A, B)=\left[\begin{array}{lllll}
0 & 0 & 0 & 0 & 1 \\
0 & 0 & 0 & 0 & 1 \\
0 & 0 & 0 & 0 & 1 \\
1 & 1 & 0 & 0 & 1 \\
0 & 0 & 0 & 0 & 1
\end{array}\right] \vee\left[\begin{array}{lllll}
0 & 0 & 0 & 1 & 0 \\
0 & 0 & 0 & 1 & 0 \\
0 & 0 & 0 & 0 & 0 \\
0 & 0 & 0 & 0 & 0 \\
1 & 1 & 1 & 1 & 1
\end{array}\right]
$$

3. Meet:

(1) Meet at vertex: There are $(1+2+4) \times 2=14$ body/line meet-at-vertex relations that can be realized based on the 25IM.

$$
\begin{gathered}
R_{25 I M}(A, B)=\left[\begin{array}{lllll}
1 & 0 & 0 & 0 & 1 \\
0 & 0 & 0 & 0 & 1 \\
0 & 0 & 0 & 0 & 1 \\
0 & 0 & 0 & 0 & 1 \\
1 & 1 & 0 & 0 & 1
\end{array}\right] \vee\left[\begin{array}{lllll}
* & 0 & 0 & 0 & 1 \\
1 & 0 & 0 & 0 & 1 \\
0 & 0 & 0 & 0 & 1 \\
0 & 0 & 0 & 0 & 1 \\
1 & 1 & 0 & 0 & 1
\end{array}\right] \vee\left[\begin{array}{ccccc}
* & 0 & 0 & 0 & 1 \\
* & 0 & 0 & 0 & 1 \\
1 & 0 & 0 & 0 & 1 \\
0 & 0 & 0 & 0 & 1 \\
1 & 1 & 0 & 0 & 1
\end{array}\right] \\
\vee \\
\vee\left[\begin{array}{lllll}
* & * & 1 & 0 & 1 \\
0 & 0 & 0 & 0 & 1 \\
0 & 0 & 0 & 0 & 0 \\
0 & 0 & 0 & 0 & 0 \\
1 & 1 & 1 & 1 & 1
\end{array}\right] \vee\left[\begin{array}{lllll}
* & 1 & 0 & 0 & 1 \\
0 & 0 & 0 & 0 & 1 \\
0 & 0 & 0 & 0 & 0 \\
0 & 0 & 0 & 0 & 0 \\
1 & 1 & 1 & 1 & 1
\end{array}\right] \vee\left[\begin{array}{lllll}
1 & 0 & 0 & 0 & 1 \\
0 & 0 & 0 & 0 & 1 \\
0 & 0 & 0 & 0 & 0 \\
0 & 0 & 0 & 0 & 0 \\
1 & 1 & 1 & 1 & 1
\end{array}\right]
\end{gathered}
$$

(2) Meet at edge: There are $\left(2^{3}+2^{5}\right) \times 2=80$ body/line meet-at-edge relations that can be realized based on the 25IM.

$$
R_{25 I M}(A, B)=\left[\begin{array}{ccccc}
* & * & 0 & 0 & 1 \\
* & 1 & 0 & 0 & 1 \\
0 & 0 & 0 & 0 & 1 \\
0 & 0 & 0 & 0 & 1 \\
1 & 1 & 0 & 0 & 1
\end{array}\right] \vee\left[\begin{array}{lllll}
* & * & 0 & 0 & 1 \\
* & * & 0 & 0 & 1 \\
* & 1 & 0 & 0 & 1 \\
0 & 0 & 0 & 0 & 1 \\
1 & 1 & 0 & 0 & 1
\end{array}\right] \vee\left[\begin{array}{ccccc}
* & * & 0 & 0 & 1 \\
* & 1 & 0 & 0 & 1 \\
0 & 0 & 0 & 0 & 0 \\
0 & 0 & 0 & 0 & 0 \\
1 & 1 & 1 & 1 & 1
\end{array}\right] \vee\left[\begin{array}{ccccc}
* & * & * & 0 & 1 \\
* & * & 1 & 0 & 1 \\
0 & 0 & 0 & 0 & 0 \\
0 & 0 & 0 & 0 & 0 \\
1 & 1 & 1 & 1 & 1
\end{array}\right],
$$

Therefore, there are $14+80=94$ body/line meet relations that can be realized based on the 25IM.

4. Cover: The body/line cover at edge relations are included in body/line meet-at-edge relations. Thus, only the expressions of body/line cover-at-vertex relations are listed here. There are $(1+2+4) \times 2=14$ body/line cover relations that can be realized based on the 25IM.

$$
\begin{gathered}
R_{25 I M}(A, B)=\left[\begin{array}{lllll}
1 & 0 & 0 & 0 & 1 \\
0 & 0 & 0 & 0 & 1 \\
0 & 0 & 0 & 0 & 1 \\
1 & 1 & 0 & 0 & 1 \\
0 & 0 & 0 & 0 & 1
\end{array}\right] \vee\left[\begin{array}{lllll}
* & 0 & 0 & 0 & 1 \\
1 & 0 & 0 & 0 & 1 \\
0 & 0 & 0 & 0 & 1 \\
1 & 1 & 0 & 0 & 1 \\
0 & 0 & 0 & 0 & 1
\end{array}\right] \vee\left[\begin{array}{lllll}
* & 0 & 0 & 0 & 1 \\
* & 0 & 0 & 0 & 1 \\
1 & 0 & 0 & 0 & 1 \\
1 & 1 & 0 & 0 & 1 \\
0 & 0 & 0 & 0 & 1
\end{array}\right] \\
\vee \\
\vee\left[\begin{array}{lllll}
1 & 0 & 0 & 1 & 0 \\
0 & 0 & 0 & 1 & 0 \\
0 & 0 & 0 & 0 & 0 \\
0 & 0 & 0 & 0 & 0 \\
1 & 1 & 1 & 1 & 1
\end{array}\right] \vee\left[\begin{array}{lllll}
* & 1 & 0 & 1 & 0 \\
0 & 0 & 0 & 1 & 0 \\
0 & 0 & 0 & 0 & 0 \\
0 & 0 & 0 & 0 & 0 \\
1 & 1 & 1 & 1 & 1
\end{array}\right] \vee\left[\begin{array}{lllll}
* & * & 1 & 1 & 0 \\
0 & 0 & 0 & 1 & 0 \\
0 & 0 & 0 & 0 & 0 \\
0 & 0 & 0 & 0 & 0 \\
1 & 1 & 1 & 1 & 1
\end{array}\right],
\end{gathered}
$$


5. Overlap: There are $2^{7} \times 2=256$ body/line overlap relations that can be realized based on the 25IM.

$$
R_{25 I M}(A, B)=\left[\begin{array}{lllll}
* & * & 0 & 0 & 1 \\
* & * & 0 & 0 & 1 \\
* & * & 0 & 0 & 1 \\
* & 1 & 0 & 0 & 1 \\
1 & 1 & 1 & 0 & 1
\end{array}\right] \vee\left[\begin{array}{lllll}
* & * & * & * & 1 \\
* & * & * & 1 & 1 \\
0 & 0 & 0 & 0 & 1 \\
0 & 0 & 0 & 0 & 0 \\
1 & 1 & 1 & 1 & 1
\end{array}\right]
$$

Therefore, there are $2+2+94+14+256=368$ body/line relations that can be realized based on the 25IM.

\subsection{Body/Point Relations}

There are three kinds of body/point topological relations: disjoint, contain, and meet. According to the definition above, point B only has vertex and exterior but no edge, face, and interior. Therefore, the intersections of all A's topological part and B's edge/face/interior are empty.

1. Disjoint: There are two body/point disjoint relations that can be realized based on the 25IM.

$$
R_{25 I M}(A, B)=\left[\begin{array}{lllll}
0 & 0 & 0 & 0 & 1 \\
0 & 0 & 0 & 0 & 1 \\
0 & 0 & 0 & 0 & 1 \\
0 & 0 & 0 & 0 & 1 \\
1 & 0 & 0 & 0 & 1
\end{array}\right] \vee\left[\begin{array}{lllll}
0 & 0 & 0 & 0 & 1 \\
0 & 0 & 0 & 0 & 0 \\
0 & 0 & 0 & 0 & 0 \\
0 & 0 & 0 & 0 & 0 \\
1 & 1 & 1 & 1 & 1
\end{array}\right]
$$

2. Contain: There are two body/point contain relations that can be realized based on the 25IM.

$$
R_{25 I M}(A, B)=\left[\begin{array}{ccccc}
0 & 0 & 0 & 0 & 1 \\
0 & 0 & 0 & 0 & 1 \\
0 & 0 & 0 & 0 & 1 \\
1 & 0 & 0 & 0 & 1 \\
0 & 0 & 0 & 0 & 1
\end{array}\right]
$$

3. Meet: The body/point meet relations mean that point B is on body A's vertex, edge, or face. There are six body/point meet relations that can be realized based on the 25IM.

$$
\begin{gathered}
R_{25 I M}(A, B)=\left[\begin{array}{lllll}
1 & 0 & 0 & 0 & 1 \\
0 & 0 & 0 & 0 & 1 \\
0 & 0 & 0 & 0 & 1 \\
0 & 0 & 0 & 0 & 1 \\
1 & 0 & 0 & 0 & 1
\end{array}\right] \vee\left[\begin{array}{lllll}
0 & 0 & 0 & 0 & 1 \\
1 & 0 & 0 & 0 & 1 \\
0 & 0 & 0 & 0 & 1 \\
0 & 0 & 0 & 0 & 1 \\
1 & 0 & 0 & 0 & 1
\end{array}\right] \vee\left[\begin{array}{lllll}
0 & 0 & 0 & 0 & 1 \\
0 & 0 & 0 & 0 & 1 \\
1 & 0 & 0 & 0 & 1 \\
0 & 0 & 0 & 0 & 1 \\
1 & 0 & 0 & 0 & 1
\end{array}\right] \\
\vee \\
\vee \\
\left.\qquad \begin{array}{lllll}
1 & 0 & 0 & 0 & 1 \\
0 & 0 & 0 & 0 & 0 \\
0 & 0 & 0 & 0 & 0 \\
0 & 0 & 0 & 0 & 0 \\
1 & 1 & 1 & 1 & 1
\end{array}\right] \vee\left[\begin{array}{lllll}
0 & 1 & 0 & 0 & 1 \\
0 & 0 & 0 & 0 & 0 \\
0 & 0 & 0 & 0 & 0 \\
0 & 0 & 0 & 0 & 0 \\
1 & 1 & 1 & 1 & 1
\end{array}\right] \vee\left[\begin{array}{lllll}
0 & 0 & 1 & 0 & 1 \\
0 & 0 & 0 & 0 & 0 \\
0 & 0 & 0 & 0 & 0 \\
0 & 0 & 0 & 0 & 0 \\
1 & 1 & 1 & 1 & 1
\end{array}\right]
\end{gathered}
$$

Therefore, there are $2+2+6=10$ body/point relations that can be realized based on the 25IM. 


\subsection{Region/Region Relations}

There are six kinds of region/region topological relations: disjoint, contain, equal, meet, cover, and overlap. Region A and region B have no interior. Therefore, the intersections of all A's topological part and B's interior are empty, and the intersections of all B's topological part and A's interior are empty.

1. Disjoint: There is only one region/region disjoint relation that can be realized based on the 25IM.

$$
R_{25 I M}(A, B)=\left[\begin{array}{lllll}
0 & 0 & 0 & 0 & 1 \\
0 & 0 & 0 & 0 & 1 \\
0 & 0 & 0 & 0 & 1 \\
0 & 0 & 0 & 0 & 0 \\
1 & 1 & 1 & 0 & 1
\end{array}\right]
$$

2. Contain: There are two region/region contain relations that can be realized based on the 25IM.

$$
R_{25 I M}(A, B)=\left[\begin{array}{lllll}
0 & 0 & 0 & 0 & 1 \\
0 & 0 & 0 & 0 & 1 \\
1 & 1 & 1 & 0 & 1 \\
0 & 0 & 0 & 0 & 0 \\
0 & 0 & 0 & 0 & 1
\end{array}\right] \vee\left[\begin{array}{lllll}
0 & 0 & 1 & 0 & 0 \\
0 & 0 & 1 & 0 & 0 \\
0 & 0 & 1 & 0 & 0 \\
0 & 0 & 0 & 0 & 0 \\
1 & 1 & 1 & 0 & 1
\end{array}\right]
$$

3. Equal: There is only one region/region equal relation that can be realized based on the 25IM.

$$
R_{25 I M}(A, B)=\left[\begin{array}{lllll}
1 & 0 & 0 & 0 & 0 \\
0 & 1 & 0 & 0 & 0 \\
0 & 0 & 1 & 0 & 0 \\
0 & 0 & 0 & 0 & 0 \\
0 & 0 & 0 & 0 & 1
\end{array}\right]
$$

4. Meet:

(1) Meet at vertex: There are $1+2+4+4+2=13$ region/region meet-at-vertex relations that can be realized based on the 25IM.

$$
\begin{aligned}
R_{25 I M}(A, B)= & {\left[\begin{array}{lllll}
1 & 0 & 0 & 0 & 1 \\
0 & 0 & 0 & 0 & 1 \\
0 & 0 & 0 & 0 & 1 \\
0 & 0 & 0 & 0 & 0 \\
1 & 1 & 1 & 0 & 1
\end{array}\right] \vee\left[\begin{array}{lllll}
* & 0 & 0 & 0 & 1 \\
1 & 0 & 0 & 0 & 1 \\
0 & 0 & 0 & 0 & 1 \\
0 & 0 & 0 & 0 & 0 \\
1 & 1 & 1 & 0 & 1
\end{array}\right] \vee\left[\begin{array}{ccccc}
* & 0 & 0 & 0 & 1 \\
* & 0 & 0 & 0 & 1 \\
1 & 0 & 0 & 0 & 1 \\
0 & 0 & 0 & 0 & 0 \\
1 & 1 & 1 & 0 & 1
\end{array}\right] } \\
& \vee\left[\begin{array}{lllll}
* & * & 1 & 0 & 1 \\
0 & 0 & 0 & 0 & 1 \\
0 & 0 & 0 & 0 & 1 \\
0 & 0 & 0 & 0 & 0 \\
1 & 1 & 1 & 0 & 1
\end{array}\right] \vee\left[\begin{array}{lllll}
* & 1 & 0 & 0 & 1 \\
0 & 0 & 0 & 0 & 1 \\
0 & 0 & 0 & 0 & 1 \\
0 & 0 & 0 & 0 & 0 \\
1 & 1 & 1 & 0 & 1
\end{array}\right],
\end{aligned}
$$


(2) Meet at edge: There are $2^{3}+2^{7}+2^{6}=200$ region/region meet-at-edge relations that can be realized based on the 25IM.

$$
R_{25 I M}(A, B)=\left[\begin{array}{ccccc}
* & * & 0 & 0 & 1 \\
* & 1 & 0 & 0 & 1 \\
0 & 0 & 0 & 0 & 1 \\
0 & 0 & 0 & 0 & 0 \\
1 & 1 & 1 & 0 & 1
\end{array}\right] \vee\left[\begin{array}{lllll}
* & * & * & 0 & 1 \\
* & * & * & 0 & 1 \\
* & 1 & 0 & 0 & 1 \\
0 & 0 & 0 & 0 & 0 \\
1 & 1 & 1 & 0 & 1
\end{array}\right] \vee\left[\begin{array}{ccccc}
* & * & * & 0 & 1 \\
* & * & 1 & 0 & 1 \\
* & 0 & 0 & 0 & 1 \\
0 & 0 & 0 & 0 & 0 \\
1 & 1 & 1 & 0 & 1
\end{array}\right]
$$

Therefore, there are $13+200=213$ region/region meet relations that can be realized based on the 25IM.

5. Cover: If region $A$ covers region $B$, then $A$ and $B$ are on the same plane.

(1) Cover at vertex: There are $(4+2) \times 2=12$ region/region cover-at-vertex relations that can be realized based on the 25IM.

$$
R_{25 I M}(A, B)=\left[\begin{array}{lllll}
1 & 0 & 0 & 0 & 1 \\
* & 0 & 0 & 0 & 1 \\
* & 1 & 1 & 0 & 1 \\
0 & 0 & 0 & 0 & 0 \\
0 & 0 & 0 & 0 & 1
\end{array}\right] \vee\left[\begin{array}{lllll}
0 & 0 & 0 & 0 & 1 \\
1 & 0 & 0 & 0 & 1 \\
* & 1 & 1 & 0 & 1 \\
0 & 0 & 0 & 0 & 0 \\
0 & 0 & 0 & 0 & 1
\end{array}\right] \vee\left[\begin{array}{lllll}
0 & 1 & * & 0 & 0 \\
0 & 0 & 1 & 0 & 0 \\
0 & 0 & 1 & 0 & 0 \\
0 & 0 & 0 & 0 & 0 \\
1 & 1 & 1 & 0 & 1
\end{array}\right] \vee\left[\begin{array}{ccccc}
1 & * & * & 0 & 0 \\
0 & 0 & 1 & 0 & 0 \\
0 & 0 & 1 & 0 & 0 \\
0 & 0 & 0 & 0 & 0 \\
1 & 1 & 1 & 0 & 1
\end{array}\right],
$$

(2) Cover at edge: There are $(4+2) \times 2=12$ region/region cover-at-edge relations that can be realized based on the 25IM.

$$
R_{25 I M}(A, B)=\left[\begin{array}{lllll}
1 & 0 & 0 & 0 & 1 \\
* & 1 & 0 & 0 & 1 \\
* & 1 & 1 & 0 & 1 \\
0 & 0 & 0 & 0 & 0 \\
0 & 0 & 0 & 0 & 1
\end{array}\right] \vee\left[\begin{array}{lllll}
0 & 0 & 0 & 0 & 1 \\
1 & 1 & 0 & 0 & 1 \\
* & 1 & 1 & 0 & 1 \\
0 & 0 & 0 & 0 & 0 \\
0 & 0 & 0 & 0 & 1
\end{array}\right] \vee\left[\begin{array}{lllll}
0 & 1 & * & 0 & 0 \\
0 & 1 & 1 & 0 & 0 \\
0 & 0 & 1 & 0 & 0 \\
0 & 0 & 0 & 0 & 0 \\
1 & 1 & 1 & 0 & 1
\end{array}\right] \vee\left[\begin{array}{lllll}
1 & * & * & 0 & 0 \\
0 & 1 & 1 & 0 & 0 \\
0 & 0 & 1 & 0 & 0 \\
0 & 0 & 0 & 0 & 0 \\
1 & 1 & 1 & 0 & 1
\end{array}\right],
$$

Therefore, there are $12+12=24$ region/region cover relations that can be realized based on the 25IM.

6. Overlap: There are $2^{8}=256$ region/region overlap relations that can be realized based on the 25IM.

$$
R_{25 I M}(A, B)=\left[\begin{array}{ccccc}
* & * & * & 0 & 1 \\
* & * & * & 0 & 1 \\
* & * & 1 & 0 & 1 \\
0 & 0 & 0 & 0 & 0 \\
1 & 1 & 1 & 0 & 1
\end{array}\right]
$$

Therefore, there are $1+2+1+213+24+256=497$ region/region relations that can be realized based on the 25IM.

\subsection{Region/Line Relations}

There are five kinds of region/region topological relations: disjoint, contain, meet, cover, and overlap. Region A has no interior, and line B has no face and interior. Therefore, the intersections of all A's topological part and B's face/interior are empty, and the intersections of all B's topological part and A's interior are empty. 
1. Disjoint: There are two region/line disjoint relations that can be realized based on the 25IM.

$$
R_{25 I M}(A, B)=\left[\begin{array}{lllll}
0 & 0 & 0 & 0 & 1 \\
0 & 0 & 0 & 0 & 1 \\
0 & 0 & 0 & 0 & 1 \\
0 & 0 & 0 & 0 & 0 \\
1 & 1 & 0 & 0 & 1
\end{array}\right] \vee\left[\begin{array}{lllll}
0 & 0 & 0 & 0 & 1 \\
0 & 0 & 0 & 0 & 1 \\
0 & 0 & 0 & 0 & 0 \\
0 & 0 & 0 & 0 & 0 \\
1 & 1 & 1 & 0 & 1
\end{array}\right]
$$

2. Contain: There are two region/line contain relations that can be realized based on the 25IM.

$$
R_{25 I M}(A, B)=\left[\begin{array}{lllll}
0 & 0 & 0 & 0 & 1 \\
0 & 0 & 0 & 0 & 1 \\
1 & 1 & 0 & 0 & 1 \\
0 & 0 & 0 & 0 & 0 \\
0 & 0 & 0 & 0 & 1
\end{array}\right] \vee\left[\begin{array}{lllll}
0 & 0 & 1 & 0 & 0 \\
0 & 0 & 1 & 0 & 0 \\
0 & 0 & 0 & 0 & 0 \\
0 & 0 & 0 & 0 & 0 \\
1 & 1 & 1 & 0 & 1
\end{array}\right]
$$

3. Meet:

(1) Meet at vertex: There are $(1+2+4) \times 2=14$ region/line meet-at-vertex relations that can be realized based on the 25IM.

$$
\begin{aligned}
R_{25 I M}(A, B) & =\left[\begin{array}{lllll}
1 & 0 & 0 & 0 & 1 \\
0 & 0 & 0 & 0 & 1 \\
0 & 0 & 0 & 0 & 1 \\
0 & 0 & 0 & 0 & 0 \\
1 & 1 & 0 & 0 & 1
\end{array}\right] \vee\left[\begin{array}{lllll}
* & 0 & 0 & 0 & 1 \\
1 & 0 & 0 & 0 & 1 \\
0 & 0 & 0 & 0 & 1 \\
0 & 0 & 0 & 0 & 0 \\
1 & 1 & 0 & 0 & 1
\end{array}\right] \vee\left[\begin{array}{lllll}
* & 0 & 0 & 0 & 1 \\
* & 0 & 0 & 0 & 1 \\
1 & 0 & 0 & 0 & 1 \\
0 & 0 & 0 & 0 & 0 \\
1 & 1 & 0 & 0 & 1
\end{array}\right] \\
& \vee\left[\begin{array}{lllll}
* & * & 1 & 0 & 1 \\
0 & 0 & 0 & 0 & 1 \\
0 & 0 & 0 & 0 & 0 \\
0 & 0 & 0 & 0 & 0 \\
1 & 1 & 1 & 0 & 1
\end{array}\right] \vee\left[\begin{array}{lllll}
* & 1 & 0 & 0 & 1 \\
0 & 0 & 0 & 0 & 1 \\
0 & 0 & 0 & 0 & 0 \\
0 & 0 & 0 & 0 & 0 \\
1 & 1 & 1 & 0 & 1
\end{array}\right] \vee\left[\begin{array}{llllll}
1 & 0 & 0 & 0 & 1 \\
0 & 0 & 0 & 0 & 1 \\
0 & 0 & 0 & 0 & 0 \\
0 & 0 & 0 & 0 & 0 \\
1 & 1 & 1 & 0 & 1
\end{array}\right],
\end{aligned}
$$

(2) Meet at edge: There are $2^{3} \times 2=16$ region/line meet-at-edge relations that can be realized based on the 25IM.

$$
R_{25 I M}(A, B)=\left[\begin{array}{ccccc}
* & * & 0 & 0 & 1 \\
* & 1 & 0 & 0 & 1 \\
0 & 0 & 0 & 0 & 1 \\
0 & 0 & 0 & 0 & 0 \\
1 & 1 & 0 & 0 & 1
\end{array}\right] \vee\left[\begin{array}{ccccc}
* & * & 0 & 0 & 1 \\
* & 1 & 0 & 0 & 1 \\
0 & 0 & 0 & 0 & 0 \\
0 & 0 & 0 & 0 & 0 \\
1 & 1 & 1 & 0 & 1
\end{array}\right]
$$

Therefore, there are $14+16=30$ region/line meet relations that can be realized based on the 25IM.

4. Cover: The region/line cover-at-edge relations are included in the region/line meet-at-edge relations. Thus, only the expressions of region/line cover-at-vertex relations are listed here. There are $(4+2) \times 2=12$ region/line cover relations that can be realized based on the 25IM.

$$
R_{25 I M}(A, B)=\left[\begin{array}{lllll}
1 & 0 & 0 & 0 & 1 \\
* & 0 & 0 & 0 & 1 \\
* & 1 & 0 & 0 & 1 \\
0 & 0 & 0 & 0 & 0 \\
0 & 0 & 0 & 0 & 1
\end{array}\right] \vee\left[\begin{array}{lllll}
0 & 0 & 0 & 0 & 1 \\
1 & 0 & 0 & 0 & 1 \\
* & 1 & 0 & 0 & 1 \\
0 & 0 & 0 & 0 & 0 \\
0 & 0 & 0 & 0 & 1
\end{array}\right] \vee\left[\begin{array}{lllll}
0 & 1 & * & 0 & 0 \\
0 & 0 & 1 & 0 & 0 \\
0 & 0 & 0 & 0 & 1 \\
0 & 0 & 0 & 0 & 0 \\
1 & 1 & 1 & 0 & 1
\end{array}\right] \vee\left[\begin{array}{lllll}
1 & * & * & 0 & 0 \\
0 & 0 & 1 & 0 & 0 \\
0 & 0 & 0 & 0 & 1 \\
0 & 0 & 0 & 0 & 0 \\
1 & 1 & 1 & 0 & 1
\end{array}\right]
$$


5. Overlap: There are $2^{5} \times 2=64$ region/line overlap relations that can be realized based on the 25IM.

$$
R_{25 I M}(A, B)=\left[\begin{array}{lllll}
* & * & 0 & 0 & 1 \\
* & * & 0 & 0 & 1 \\
* & 1 & 0 & 0 & 1 \\
0 & 0 & 0 & 0 & 0 \\
1 & 1 & 0 & 0 & 1
\end{array}\right] \vee\left[\begin{array}{lllll}
* & * & * & 0 & 1 \\
* & * & 1 & 0 & 1 \\
0 & 0 & 0 & 0 & 0 \\
0 & 0 & 0 & 0 & 0 \\
1 & 1 & 1 & 0 & 1
\end{array}\right]
$$

Therefore, there are $2+2+30+12+64=110$ region/line relations that can be realized based on the 25IM.

\subsection{Region/Point Relations}

There are three kinds of region/point topological relations: disjoint, contain, and meet. Region A has no interior, and point B has no edge, face, and interior. Therefore, the intersections of all A's topological part and B's edge/face/interior are empty, and the intersections of all B's topological part and A's interior are empty.

1. Disjoint: There are two region/point disjoint relations that can be realized based on the 25IM.

$$
R_{25 I M}(A, B)=\left[\begin{array}{lllll}
0 & 0 & 0 & 0 & 1 \\
0 & 0 & 0 & 0 & 1 \\
0 & 0 & 0 & 0 & 1 \\
0 & 0 & 0 & 0 & 0 \\
1 & 0 & 0 & 0 & 1
\end{array}\right] \vee\left[\begin{array}{ccccc}
0 & 0 & 0 & 0 & 1 \\
0 & 0 & 0 & 0 & 0 \\
0 & 0 & 0 & 0 & 0 \\
0 & 0 & 0 & 0 & 0 \\
1 & 1 & 1 & 0 & 1
\end{array}\right]
$$

2. Contain: There are two region/line contain relations that can be realized based on the 25IM.

$$
R_{25 I M}(A, B)=\left[\begin{array}{lllll}
0 & 0 & 0 & 0 & 1 \\
0 & 0 & 0 & 0 & 1 \\
1 & 0 & 0 & 0 & 1 \\
0 & 0 & 0 & 0 & 0 \\
0 & 0 & 0 & 0 & 1
\end{array}\right] \vee\left[\begin{array}{lllll}
0 & 0 & 1 & 0 & 0 \\
0 & 0 & 0 & 0 & 0 \\
0 & 0 & 0 & 0 & 0 \\
0 & 0 & 0 & 0 & 0 \\
1 & 1 & 1 & 0 & 1
\end{array}\right]
$$

3. Meet: A region/point meet relation means that point B is on the vertex or edge of body A. There are four body/point meet relations that can be realized based on the 25IM.

$$
R_{25 I M}(A, B)=\left[\begin{array}{lllll}
1 & 0 & 0 & 0 & 1 \\
0 & 0 & 0 & 0 & 1 \\
0 & 0 & 0 & 0 & 1 \\
0 & 0 & 0 & 0 & 0 \\
0 & 0 & 0 & 0 & 1
\end{array}\right] \vee\left[\begin{array}{lllll}
0 & 0 & 0 & 0 & 1 \\
1 & 0 & 0 & 0 & 1 \\
0 & 0 & 0 & 0 & 1 \\
0 & 0 & 0 & 0 & 0 \\
0 & 0 & 0 & 0 & 1
\end{array}\right] \vee\left[\begin{array}{lllll}
0 & 1 & 0 & 0 & 0 \\
0 & 0 & 0 & 0 & 0 \\
0 & 0 & 0 & 0 & 0 \\
0 & 0 & 0 & 0 & 0 \\
1 & 1 & 1 & 0 & 1
\end{array}\right] \vee\left[\begin{array}{lllll}
1 & 0 & 0 & 0 & 0 \\
0 & 0 & 0 & 0 & 0 \\
0 & 0 & 0 & 0 & 0 \\
0 & 0 & 0 & 0 & 0 \\
1 & 1 & 1 & 0 & 1
\end{array}\right]
$$

Therefore, there are $2+2+4=8$ region/point relations that can be realized based on the 25IM.

\subsection{Linelline relations}

There are six kinds of region/region topological relations: disjoint, contain, equal, meet, cover, and overlap. Region A and B have no face and no interior. Therefore, the intersections of all A's topological part and B's face/interior are empty, and the intersections of all B's topological part and A's face/interior are empty. 
1. Disjoint: There is only one line/line disjoint relation that can be realized based on the 25IM.

$$
R_{25 I M}(A, B)=\left[\begin{array}{ccccc}
0 & 0 & 0 & 0 & 1 \\
0 & 0 & 0 & 0 & 1 \\
0 & 0 & 0 & 0 & 0 \\
0 & 0 & 0 & 0 & 0 \\
1 & 1 & 0 & 0 & 1
\end{array}\right]
$$

2. Contain: There are two region/line contain relations that can be realized based on the 25IM.

$$
R_{25 I M}(A, B)=\left[\begin{array}{lllll}
0 & 0 & 0 & 0 & 1 \\
1 & 1 & 0 & 0 & 1 \\
0 & 0 & 0 & 0 & 0 \\
0 & 0 & 0 & 0 & 0 \\
0 & 0 & 0 & 0 & 1
\end{array}\right] \vee\left[\begin{array}{ccccc}
0 & 1 & 0 & 0 & 0 \\
0 & 1 & 0 & 0 & 0 \\
0 & 0 & 0 & 0 & 0 \\
0 & 0 & 0 & 0 & 0 \\
1 & 1 & 0 & 0 & 1
\end{array}\right]
$$

3. Equal: There is only one line/line equal relation that can be realized based on the 25IM.

$$
R_{25 I M}(A, B)=\left[\begin{array}{ccccc}
1 & 0 & 0 & 0 & 0 \\
0 & 1 & 0 & 0 & 0 \\
0 & 0 & 0 & 0 & 0 \\
0 & 0 & 0 & 0 & 0 \\
0 & 0 & 0 & 0 & 1
\end{array}\right]
$$

4. Meet: Line/line meet-at-edge relations are included in line/line overlap relations. Therefore, only line/line meet-at-vertex relations are listed here. There are $1+2+2=5$ line/line meet-at-vertex relations that can be realized based on the 25IM.

$$
R_{25 I M}(A, B)=\left[\begin{array}{lllll}
1 & 0 & 0 & 0 & 1 \\
0 & 0 & 0 & 0 & 1 \\
0 & 0 & 0 & 0 & 0 \\
0 & 0 & 0 & 0 & 0 \\
1 & 1 & 0 & 0 & 1
\end{array}\right] \vee\left[\begin{array}{ccccc}
* & 0 & 0 & 0 & 1 \\
1 & 0 & 0 & 0 & 1 \\
0 & 0 & 0 & 0 & 0 \\
0 & 0 & 0 & 0 & 0 \\
1 & 1 & 0 & 0 & 1
\end{array}\right] \vee\left[\begin{array}{ccccc}
* & 1 & 0 & 0 & 1 \\
0 & 0 & 0 & 0 & 1 \\
0 & 0 & 0 & 0 & 0 \\
0 & 0 & 0 & 0 & 0 \\
1 & 1 & 0 & 0 & 1
\end{array}\right]
$$

5. Cover: There are two line/line cover relations that can be realized based on the 25IM.

$$
R_{25 I M}(A, B)=\left[\begin{array}{lllll}
1 & 0 & 0 & 0 & 1 \\
1 & 1 & 0 & 0 & 1 \\
0 & 0 & 0 & 0 & 0 \\
0 & 0 & 0 & 0 & 0 \\
0 & 0 & 0 & 0 & 1
\end{array}\right] \vee\left[\begin{array}{lllll}
1 & 1 & 0 & 0 & 0 \\
0 & 1 & 0 & 0 & 0 \\
0 & 0 & 0 & 0 & 0 \\
0 & 0 & 0 & 0 & 0 \\
1 & 1 & 0 & 0 & 1
\end{array}\right]
$$

6. Overlap: There are $2^{3}=8$ line/line overlap relations that can be realized based on the 25IM.

$$
R_{25 I M}(A, B)=\left[\begin{array}{ccccc}
* & * & 0 & 0 & 1 \\
* & 1 & 0 & 0 & 1 \\
0 & 0 & 0 & 0 & 0 \\
0 & 0 & 0 & 0 & 0 \\
1 & 1 & 0 & 0 & 1
\end{array}\right]
$$

Therefore, there are $1+2+1+5+2+8=19$ line/line meet relations that can be realized based on the 25IM. 


\subsection{Line/Point Relations}

There are three kinds of line/point topological relations: disjoint, contain, and meet. Line A has no face and interior, and point B has no edge, face, and interior. Therefore, the intersections of all A's topological part and B's edge/face/interior are empty, and the intersections of all B's topological part and A's face/interior are empty.

1. Disjoint: There are two line/point disjoint relations that can be realized based on the 25IM.

$$
R_{25 I M}(A, B)=\left[\begin{array}{lllll}
0 & 0 & 0 & 0 & 1 \\
0 & 0 & 0 & 0 & 1 \\
0 & 0 & 0 & 0 & 0 \\
0 & 0 & 0 & 0 & 0 \\
1 & 0 & 0 & 0 & 1
\end{array}\right] \vee\left[\begin{array}{lllll}
0 & 0 & 0 & 0 & 1 \\
0 & 0 & 0 & 0 & 0 \\
0 & 0 & 0 & 0 & 0 \\
0 & 0 & 0 & 0 & 0 \\
1 & 1 & 0 & 0 & 1
\end{array}\right]
$$

2. Contain: Line A contains point B when B is on A's edge. There are two line/point contain relations that can be realized based on the 25IM.

$$
R_{25 I M}(A, B)=\left[\begin{array}{lllll}
0 & 0 & 0 & 0 & 1 \\
1 & 0 & 0 & 0 & 1 \\
0 & 0 & 0 & 0 & 0 \\
0 & 0 & 0 & 0 & 0 \\
1 & 0 & 0 & 0 & 1
\end{array}\right] \vee\left[\begin{array}{lllll}
0 & 1 & 0 & 0 & 1 \\
0 & 0 & 0 & 0 & 0 \\
0 & 0 & 0 & 0 & 0 \\
0 & 0 & 0 & 0 & 0 \\
1 & 1 & 0 & 0 & 1
\end{array}\right]
$$

3. Meet: Line A meets point B when B is the vertex of A. There are two line/point meet relations that can be realized based on the 25IM.

$$
R_{25 I M}(A, B)=\left[\begin{array}{lllll}
1 & 0 & 0 & 0 & 1 \\
0 & 0 & 0 & 0 & 1 \\
0 & 0 & 0 & 0 & 0 \\
0 & 0 & 0 & 0 & 0 \\
1 & 0 & 0 & 0 & 1
\end{array}\right] \vee\left[\begin{array}{lllll}
1 & 0 & 0 & 0 & 1 \\
0 & 0 & 0 & 0 & 0 \\
0 & 0 & 0 & 0 & 0 \\
0 & 0 & 0 & 0 & 0 \\
1 & 1 & 0 & 0 & 1
\end{array}\right]
$$

Therefore, there are $2+2+2=6$ line/point relations that can be realized based on the 25IM.

\subsection{Point/Point Relations}

There are two kinds of point/point topological relations: disjoint and equal.

1. Disjoint: There is only one point/point disjoint relation that can be realized based on the 25IM.

$$
R_{25 I M}(A, B)=\left[\begin{array}{lllll}
0 & 0 & 0 & 0 & 1 \\
0 & 0 & 0 & 0 & 0 \\
0 & 0 & 0 & 0 & 0 \\
0 & 0 & 0 & 0 & 0 \\
1 & 0 & 0 & 0 & 1
\end{array}\right]
$$

2. Equal: There is only one point/point equal relation that can be realized based on the 25IM.

$$
R_{25 I M}(A, B)=\left[\begin{array}{lllll}
1 & 0 & 0 & 0 & 0 \\
0 & 0 & 0 & 0 & 0 \\
0 & 0 & 0 & 0 & 0 \\
0 & 0 & 0 & 0 & 0 \\
0 & 0 & 0 & 0 & 1
\end{array}\right]
$$

Therefore, there are two line/point relations that can be realized based on the 25IM. 


\section{Discussion}

The 9IM and the DE-9IM are chosen to be compared with the 25IM (Table 1) because they are the most commonly used models to express topological relations between two simple spatial objects. They can only handle simple objects and cannot handle complex objects. The models designed for complex objects with holes (e.g., 9+IM) are not discussed here because only simple objects are considered in this paper. Only some typical relations (mostly body/body relations) are compared because there are a large number of topological relations and some relations are similar.

The 9IM has only one expression for each disjoint, contain, equal, meet, cover, and overlap and, thus, cannot reflect the dimensions of the two spatial objects. The topological relations between spatial objects with different dimensions in 3-D space are easily confused in the 9IM (e.g., body/body relations and region/region relations) because they have the same expressions. The DE-9IM considers the dimensions of the intersections and, thus, can distinguish more topological relations than the 9IM. The DE-9IM only records the highest dimensions of intersections and cannot distinguish whether there are intersections of lower dimensions. For example, when two bodies meet at a face, the DE-9IM cannot distinguish whether they share a common vertex. Moreover, both the 9IM and the DE-9IM cannot distinguish whether two objects meet/cover at a vertex, edge, or face.

Table 1. The 9IM, DE-9IM, and 25IM expressions of body/body relations.

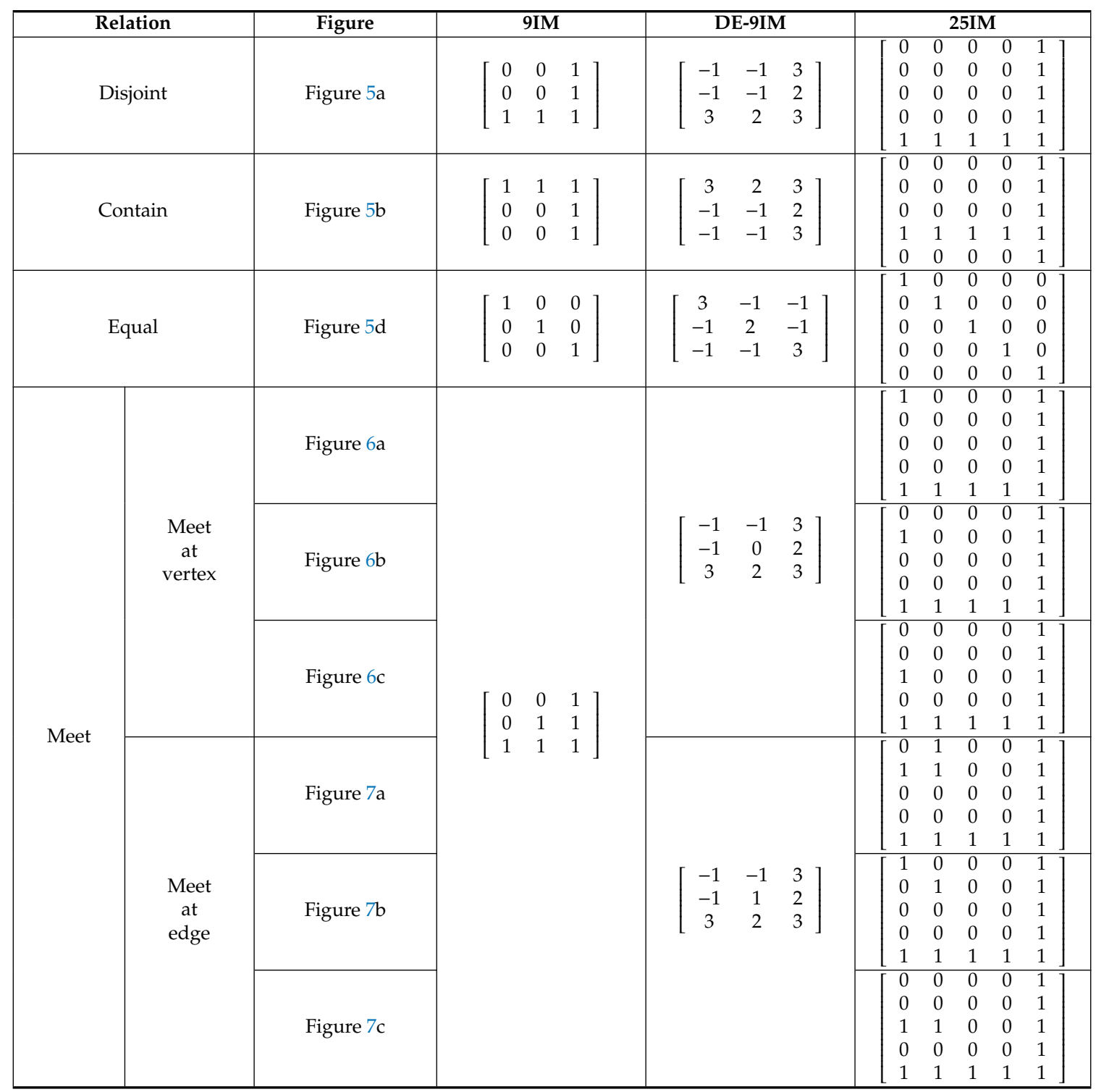


Table 1. Cont.

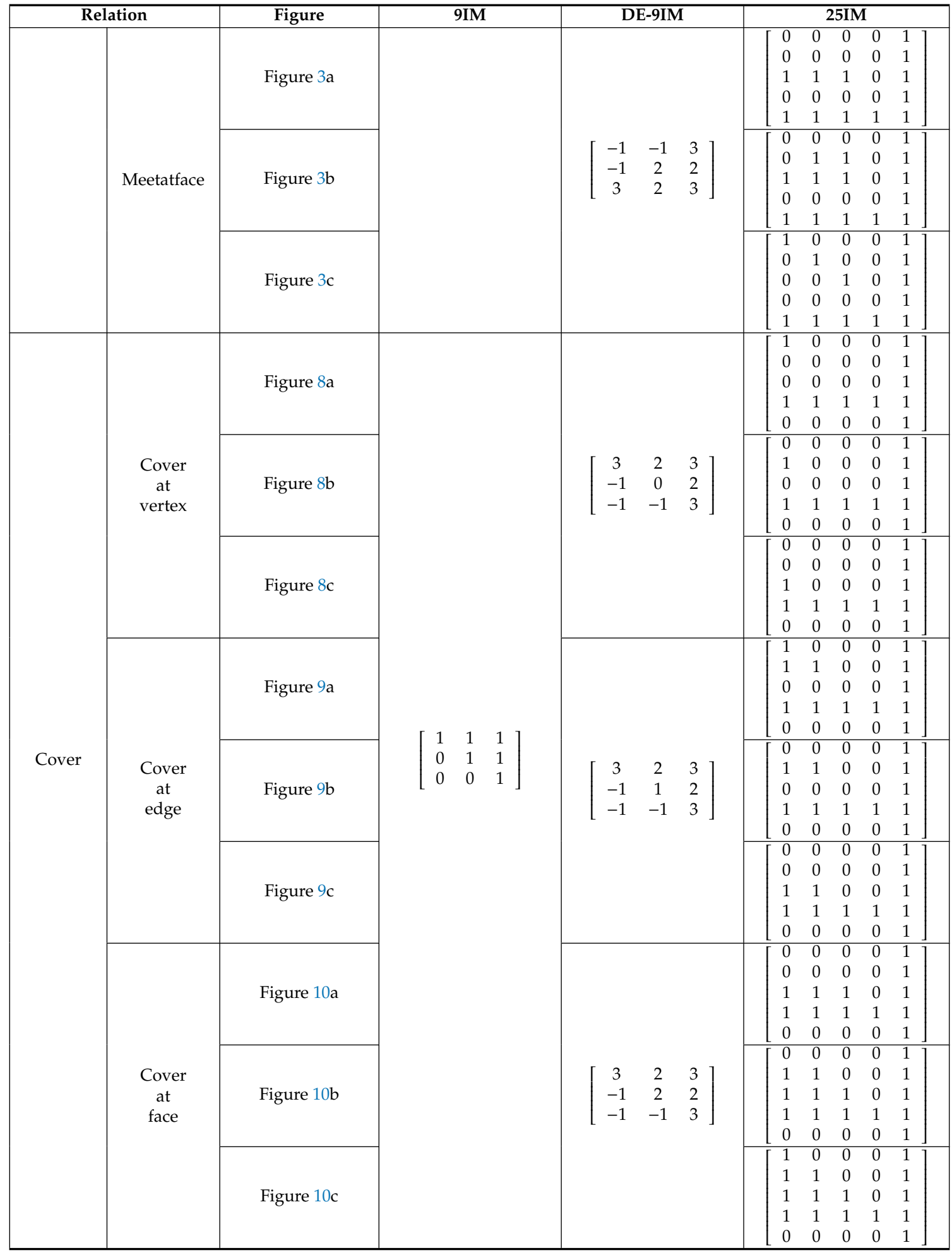


Table 1. Cont.

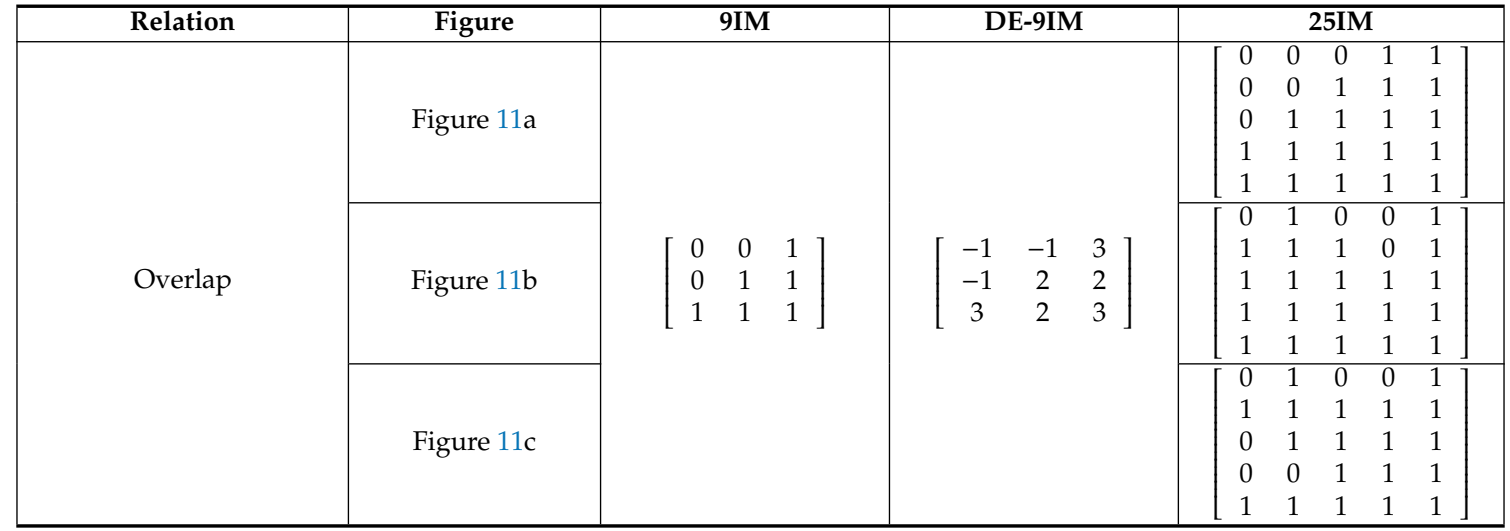

The 25IM subdivides the topological part "boundary" into vertex, edge, and face and, thus, can distinguish the topological relations in detail. The 25IM can distinguish which graphic primitives of the two spatial objects are intersected. Furthermore, the types of the two spatial objects are obvious in the 25IM. The corresponding topological part of $A$ is nonexistent when all values of a row are empty, and the corresponding topological part of $B$ is nonexistent when all values of a column are empty.

The 25IM are more complex but also more expressive than the 9IM and the DE-9IM for representing topological relations between simple spatial objects in 3-D space. The 25IM subdivided the relations expressed by the same matrix in 9IM and DE-9IM. For example, the topological relations in Figures 3 and 6, Figures 7-11 are obviously different, but they have the same 9IM and DE-9IM expressions as shown in Table 1.

\section{Conclusions}

This paper proposes a 25IM for representing topological relations between simple spatial objects in $3=\mathrm{D}$ Space. The main contributions of this paper are as follows:

1. The 25IM refines the 9IM by subdividing the topological part "boundary" into vertex, edge, and face.

2. The classification of topological relations is simplified by merging contain/inside and cover/coveredby because " $A$ is covered by $B$ " is equal to " $B$ covers $A$ " and " $A$ is inside $B$ " is equal to "B contains $A$ ". The matrix of " $A$ is covered by $B$ " is the transposed matrix of "A covers $B$ ", and the matrix of "A is inside B" is the transposed matrix of "A contains B".

3. The 25IM expressions of topological relations that exist in reality are listed. The 25IM can totally distinguish 6259 relations that exist in the real world:

- 2651 body/body relations (one disjoint relation, two contain relations, one equal relation, 461 meet relations, 138 cover relations, and 2048 overlap relations),

- 2588 body/region relations (two disjoint relations, two contain relations, 910 meet relations, 138 cover relations, and 1536 overlap relations),

- 368 body/line relations (two disjoint relations, two contain relations, 94 meet relations, 14 cover relations, and 256 overlap relations),

- 10 body/point relations (two disjoint relations, two contain relations, and six meet relations),

- 497 region/region relation (one disjoint relation, two contain relations, one equal relation, 213 meet relations, 24 cover relations, and 256 overlap relations),

- 110 region/line relations (two disjoint relations, two contain relations, 30 meet relations, 12 cover relations, and 64 overlap relations),

- 8 region/point relations (two disjoint relations, two contain relations, and four meet relations), 
- 19 line/line relations (one disjoint relation, two contain relations, one equal relation, five meet relations, two cover relations, and eight overlap relations),

- 6 line/point relations (two disjoint relations, two contain relations, and two meet relations) and

- two point/point relations (one disjoint relation and one equal relation).

4. The 25IM is demonstrated to be more expressive than the 9IM and the DE-9IM, especially in distinguishing the details of situations of meet, cover, and overlap (e.g., two bodies meet/cover at a vertex, edge, or face).

There are a large number of topological relations that can be distinguished by the 25IM. Because some relations are similar, only the diagrams of some typical relations are shown. Only the topological relations between simple spatial objects in 3-D space have been considered in this paper. However, the topological relations between complex bodies with holes are also important in applications, and they will be discussed in our future work. In addition, the implementations of the 25IM involving the topological calculation and topological reasoning will be discussed in our future work.

Author Contributions: Conceptualization, Mengyun Zhou; methodology, Mengyun Zhou; validation, Mengyun Zhou and Qingfeng Guan; resources, Qingfeng Guan; writing_-original draft preparation, Mengyun Zhou; writing - review and editing, Mengyun Zhou and Qingfeng Guan; funding acquisition, Mengyun Zhou.

Funding: This research was funded by the China Postdoctoral Science Foundation, grant number 2017M612532.

Acknowledgments: The comments from the anonymous reviewers and editors are appreciated.

Conflicts of Interest: The authors declare no conflict of interest.

\section{References}

1. Sharma, J. Integrated Spatial Reasoning in Geographic Information Systems: Combining Topology and Direction. Ph.D. Thesis, University of Maine, Orono, ME, USA, 1996.

2. Worboys, M.F.; Bofakos, P. A canonical model for a class of areal spatial objects. In Proceedings of the International Symposium on Spatial Databases, Singapore, $23-25$ June 1993; pp. 36-52.

3. Egenhofer, M.J.; Mark, D.M. Naive geography. In Proceedings of the International Conference on Spatial Information Theory, Semmering, Austria, 21-23 September 1995; pp. 1-15.

4. Dube, M.P. Topological augmentation: A step forward for qualitative partition reasoning. J. Spat. Inf. Sci. 2017, 2017, 1-29. [CrossRef]

5. Egenhofer, M.J.; Franzosa, R.D. Point-set topological spatial relations. Int. J. Geogr. Inf. Syst. 1991, 5, 161-174. [CrossRef]

6. Egenhofer, M.J.; Herring, J. Categorizing Binary Topological Relations between Regions, Lines, and Points in Geographic Databases; University of Maine: Orono, ME, USA, 1991; p. 28.

7. Clementini, E.; Di Felice, P.; Van Oosterom, P. A small set of formal topological relationships suitable for end-user interaction. In Proceedings of the International Symposium on Spatial Databases, Singapore, 23-25 June 1993; pp. 277-295.

8. Deng, M.; Cheng, T.; Chen, X.; Li, Z. Multi-level topological relations between spatial regions based upon topological invariants. GeoInformatica 2007, 11, 239-267. [CrossRef]

9. Liu, K.; Shi, W. Extended model of topological relations between spatial objects in geographic information systems. Int. J. Appl. Earth Observ. Geoinf. 2007, 9, 264-275. [CrossRef]

10. Kurata, Y. The 9+-intersection: A universal framework for modeling topological relations. In Proceedings of the International Conference on Geographic Information Science, Park City, UT, USA, 23-26 September 2008; pp. 181-198.

11. Alboody, A.; Sedes, F.; Inglada, J. Modeling topological relations between uncertain spatial regions in geo-spatial databases: Uncertain intersection and difference topological model. In Proceedings of the 2010 Second International Conference on Advances in Databases, Knowledge, and Data Applications, Menuires, France, 11-16 April 2010; pp. 7-15.

12. Shen, J.; Zhou, T.; Chen, M. A 27-intersection model for representing detailed topological relations between spatial objects in two-dimensional space. ISPRS Int. J. Geo-Inf. 2017, 6, 37. [CrossRef] 
13. Shen, J.; Zhang, L.; Chen, M. Topological relations between spherical spatial regions with holes. Int. J. Digit. Earth 2018, 1-28. [CrossRef]

14. Kurata, Y. From three-dimensional topological relations to contact relations. In Developments in 3D Geo-Information Sciences; Springer: Berlin/Heidelberg, Germany, 2010; pp. 123-142.

15. Zlatanova, S. On 3D topological relationships. In Proceedings of the 11th International Workshop on Database and Expert Systems Applications, London, UK, 4-8 September 2000; pp. 913-919.

(C) 2019 by the authors. Licensee MDPI, Basel, Switzerland. This article is an open access article distributed under the terms and conditions of the Creative Commons Attribution (CC BY) license (http://creativecommons.org/licenses/by/4.0/). 